\title{
From well-quasi-ordered sets to better-quasi-ordered sets
}

\author{
Maurice Pouzet * \\ PCS, Université Claude-Bernard Lyon1, Domaine de Gerland -bât. \\ Recherche [B], \\ 50 avenue Tony-Garnier, F69365 Lyon cedex 07, France \\ pouzet@univ-lyon1.fr \\ Norbert Sauer ${ }^{\dagger}$ \\ Department of Mathematics and Statistics, The University of Calgary, \\ Calgary, T2N1N4, Alberta, Canada \\ nsauer@math . ucalgary . ca
}

Submitted: Jul 17, 2005; Accepted: Oct 18, 2006; Published: Nov 6, 2006

Mathematics Subject Classification: 06A06, 06A07

\begin{abstract}
We consider conditions which force a well-quasi-ordered poset (wqo) to be betterquasi-ordered (bqo). In particular we obtain that if a poset $P$ is wqo and the set $S_{\omega}(P)$ of strictly increasing sequences of elements of $P$ is bqo under domination, then $P$ is bqo. As a consequence, we get the same conclusion if $S_{\omega}(P)$ is replaced by $\mathcal{J} \neg(P)$, the collection of non-principal ideals of $P$, or by $A M(P)$, the collection of maximal antichains of $P$ ordered by domination. It then follows that an interval order which is wqo is in fact bqo.
\end{abstract}

Key words: poset, ideal, antichain, domination quasi-order, interval-order, barrier, well-quasiordered set, better-quasi-ordered set.

*Supported by Intas. Research done while the author visited the Math. Dept. of U of C, in spring 2005, under a joint agreement between the two universities; the support provided is gratefully acknowledged.

†'Supported by NSERC of Canada Grant \# 691325 


\section{Introduction and presentation of the results}

\subsection{How to read this paper}

Section 7 contains a collection of definitions, notations and basic facts. The specialist reader should be able to read the paper with only occasional use of Section 7 to check up on some notation. Section 7 provides readers which are not very familiar with the topic of the paper with some background, definitions and simple derivations from those definitions. Such readers will have to peruse Section 7 frequently.

The paper is organized as follows. Section 2 provides the basics behind the notion of bqo posets and develops the technical tools we need to work with barriers and concludes with the proof of a result about $\alpha$-bqo's from which Theorem 1.1 follows. We present some topological properties of ideals in Section 3 and discuss minimal type posets in Section 4. The proof of Theorem 1.7 is contained in Section 5. In Section 6 we present constructions involving maximal antichains of prescribed size.

\section{$1.2 \quad$ Acknowledgments}

We are grateful to the referee of this paper for an extraordinary careful reading and many useful suggestions which contributed substantially to the final version of this paper.

\subsection{Background}

Since their introduction by G.Higman [10], well-quasi-ordered sets (wqo) have played an important role in several areas of mathematics: algebra (embeddability of free algebras in skew-fields, elimination orderings), set theory and logic (comparison of chains, termination of rewriting systems, decision problems), analysis (asymptotic computations, symbolic dynamic). A recent example is given by the Robertson-Seymour Theorem [25] asserting that the collection of finite graphs is well-quasi-ordered by the minor relation.

In this paper we deal with the stronger notion of better-quasi-ordered sets (bqo). Bqo posets where introduced by C. St. J. A. Nash-Williams, see [19], to prove that the class of infinite trees is wqo under topological embedding.

Better-quasi-orders enjoy several properties of well-quasi-orders. For example, finite posets are bqo. Well ordered chains are bqo, finite unions and finite products of bqo posets are bqo. The property of being bqo is preserved under restrictions and epimorphic images. Still there is a substantial difference: Better-quasi-ordered posets are preserved under the infinitary construction described in the next paragraph, but well-quasi-ordered posets are not.

A basic result due to G.Higman, see [10], asserts that a poset $P$ is wqo if and only if $\mathbf{I}(P)$, the set of initial segments of $P$, is well-founded. On the other hand, Rado [24] has produced an example of a well-founded partial order $P$ for which $\mathbf{I}(P)$ is well-founded and contains infinite antichains. The idea behind the bqo notion is to forbid this situation: $\mathbf{I}(P)$ and all its iterates, $\mathbf{I}\left(\mathbf{I}(\cdots(\mathbf{I}(P) \cdots))\right.$ up to the ordinal $\omega_{1}$, have to be well-founded and hence wqo. 
This idea is quite natural but not workable. (Proving that a two element set satisfies this property is far from being an easy task). The working definition, based upon the notion of barrier, invented by C. St. J. A. Nash-Williams, is quite involved, see [18] and [19]. Even using this working condition, it is not so easy to see wether a wqo is a bqo or not. We aim to arrive at a better understanding of bqo posets and consider two special problems to see if indeed we obtained such a better understanding.

We solved the first problem, to characterize bqo interval orders, completely, see Theorem 1.6. The second was Bonnet's problem, see Problem 1.8. We related the property of a poset to be bqo to the bqo of various posets associated to a given poset, in particular the poset of the maximal antichains under the domination order. We think that those results stand on their own but unfortunately don't seem to be strong enough to solve Bonnet's problem.

\subsection{The results}

Let $P$ be a poset.

For $X, Y \subseteq P$, let $X \leq_{\text {dom }} Y$ if for every $x \in X$ there is a $y \in Y$ with $x \leq y$; this defines a quasi-order, the domination quasi-order, on $\mathfrak{P}(P)$. Let $S_{\omega}(P)$ be the set of strictly increasing $\omega$-sequences of elements of $P$. We will prove, see Theorem 2.17 and the paragraph before it:

Theorem 1.1 If $P$ is wqo, and $\left(S_{\omega}(P) ; \leq_{d o m}\right)$ is bqo then $P$ is bqo.

Let $C \in S_{\omega}(P)$. Then $\downarrow C$ is an ideal of $P$. On the other hand if $I$ is an ideal with denumerable cofinality then $I=\downarrow C$ for some $C \in S_{\omega}(P)$.

Let $\mathcal{J}^{\neg \downarrow}(P)$ be the set of non principal ideals. Since ideals with denumerable cofinality are non-principal, we obtain from Theorem 1.1 and the property of bqo to be preserved under restrictions that:

Corollary 1.2 If $P$ is wqo and $\mathcal{J}^{\neg \downarrow}(P)$ is bqo then $P$ is bqo.

The poset $\left(S_{\omega}(P) ; \leq_{d o m}\right)$ is often more simple than the poset $P$. So for example if $P$ is finite $S_{\omega}(P)=\emptyset$. It follows trivially from Definition 2.4 that the empty poset is bqo and hence from Theorem 1.1 that finite posets are bqo. A result which is of course well known. Also:

Corollary 1.3 If $P$ is wqo and $\mathcal{J}^{\neg \downarrow}(P)$ is finite then $P$ is bqo.

Corollary 1.2 was conjectured by the first author in his thesis [21] and a proof of Corollary 1.3 given there. The proof is given in [6] Chapter 7, subsections 7.7.7 and 7.7.8. pp $217-219$.

The above considerations suggest that $S_{\omega}(P)$ corresponds to some sort of derivative. As already observed, the elements of $S_{\omega}(P)$ generate the non-principal ideals of $P$ with denumerable cofinality. As a subset of $\mathfrak{P}(P)$ equipped with the usual topology, $\mathcal{J}(P)$ is 
closed whenever $P$ is wqo. Hence, equipped with the topology induced by the topology on $\mathfrak{P}(P)$, it becomes a compact totally disconnected space $C$, see Section 3. It follows that $P$ is finite if and only if $C^{(1)}$, the first Cantor-Bendixson derivative of $C$, is empty and that $\mathcal{J}^{\neg \downarrow}(P)$ is finite if and only if the second Cantor-Bendixson derivative $C^{(2)}$ of $C$ is empty.

The space $C$ contains just one limit if and only if $\mathcal{J}^{\neg \downarrow}(P)$ is a singleton space. If the limit is $P$ itself, such a poset is called a minimal type poset. Minimal type posets occur naturally in symbolic dynamics. See section 4 for details.

Corollary 1.2 has the immediate consequence:

Corollary 1.4 If $P$ is wqo and $\mathcal{J}^{\neg \downarrow}(P)$ is a chain then $P$ is bqo.

Proof. Indeed, if $P$ is wqo then $\mathbf{I}(P)$ is well-founded. In particular $\mathcal{J}^{\neg \downarrow}(P)$ is wellfounded. If $\mathcal{J}^{\neg \downarrow}(P)$ is a chain, this is a well-ordered chain, hence a bqo. From Corollary $1.2 P$ is bqo.

Lemma 1.5 If $P$ is an interval order then $\mathcal{J}^{\neg \downarrow}(P)$ is a chain.

Proof. Let $I, J \in \mathcal{J}^{\neg \downarrow}(P)$. If $I \backslash J \neq \emptyset$ and $J \backslash I \neq \emptyset$ pick $x \in I \backslash J$ and $y \in J \backslash I$. Since $I$ is not a principal ideal then $x$ is not a maximal element in $I$, so we may pick $x^{\prime} \in I$ such that $x<x^{\prime}$. For the same reason, we may pick $y^{\prime} \in J$ such that $y<y^{\prime}$. Clearly, the poset induced on $\left\{x, x^{\prime}, y, y^{\prime}\right\}$ is a $\underline{2} \oplus \underline{2}$. But then $P$ is not an interval order.

From Corollary 1.4, this gives:

Theorem 1.6 An interval order is bqo iff it is wqo.

We will prove:

Theorem 1.7 Let $P$ be a poset. If $P$ has no infinite antichain, then the following properties are equivalent:

(i) $P$ is bqo.

(ii) $\left(P ; \leq_{\text {succ }}\right)$ is bqo.

(iii) $\left(P ; \leq_{\text {pred }}\right)$ is bqo.

(iv) $\left(P ; \leq_{\text {crit }}\right)$ is bqo.

(v) $A M(P)$ is bqo.

Theorem 1.7 turns out to be an immediate consequence of Theorem 5.5.

As indicated earlier, part of the motivation for this research was an intriguing problem due to Bonnet, see [4].

Problem 1.8 Is every wqo poset a countable union of bqo posets? 
Let $P$ be a wqo poset. If the size of the antichains of $P$ is bounded by some integer, say $m$, then from Dilworth's theorem, $P$ is the union of at most $m$ chains. Because $P$ is well founded, these chains are well ordered, hence are bqo, and $P$ is bqo as a finite union of bqo's, establishing Bonnet's conjecture in that case. This observation and item $(v)$ of Theorem 1.7 may suggest to attack Bonnet's problem using the antichains of the poset.

For each integer $m$, let $A M_{m}(P)$ be the collection of maximal antichains having size $m$ and $Q_{m}:=\bigcup A M_{m}(P)$ be the union of these maximal antichains. The partial order $P$ is the countable union of the sets $Q_{m}$. Hence the first idea to resolve Bonnet's problem would be to try to prove that the partial orders $Q_{m}$ are bqo. Note that the sizes of the antichains in $Q_{m}$ could be unbounded. Hence $Q_{m}$ might not be bqo. As an encouraging result we found wqo posets $P$ for which $Q_{m}$ is bqo for every $m$ and $A M(P)$, and hence $P$, is not bqo. Rado's poset provides an example, see Lemma 6.5.

But unfortunately, it follows from Lemma 6.4, that there is a wqo poset $P$ for which $Q_{2}$ is not bqo. Still, we feel that a more detailed investigation of the partial orders $Q_{m}$, $A M_{m}(P)$ and $A M(P)$ might give some insight into Bonnet's problem.

We can prove, see Theorem 6.3:

Theorem 1.9 Let $P$ be a poset with no infinite antichain, then $A M_{2}(P)$ is bqo if and only if $Q_{2}$ is bqo.

It follows from Corollary 6.7 that there exists a wqo poset $P$ for which $A M_{3}(P)$ is bqo but $Q_{3}$ is not bqo.

\section{Barriers and better-quasi-orders}

\section{$2.1 \quad$ Basics}

We use Nash-William's notion of bqo, see [18], and refer to Milner's exposition of bqo theory, see [16]. See Section 7 for the basic definitions.

The following result due to F.Galvin extends the partition theorem of F.P.Ramsey.

Theorem 2.1 [8] For every subset $B$ of $[\mathbb{N}]^{<\omega}$ there is an infinite subset $X$ of $\mathbb{N}$ such that either $[X]^{<\omega} \cap B=\emptyset$ or $[X]^{<\omega} \cap B$ is a block.

The theorem of Galvin implies the following result of Nash-Williams, see [16].

Theorem 2.2 (a) Every block contains a barrier.

(b) For every partition of a barrier into finitely many parts, one contains a barrier.

The partial order $\left(B, \leq_{l e x}\right)$ is the lexicographic sum of the partial orders $\left(B_{(i)}, \leq_{l e x}\right)$ :

$$
\left(B, \leq_{l e x}\right)=\sum_{i \in \mathbb{N}}\left(B_{(i)}, \leq_{l e x}\right)
$$


Let $\mathrm{T}(B)$ be the tree $\mathrm{T}(B):=\left(\left\{t: \exists s \in B\left(t \leq_{i n} s\right)\right\}, \leq_{i n}\right)$ with root $\emptyset$ and $\mathrm{T}^{d}(B)$ the dual order of $\mathrm{T}(B)$. If $\mathrm{T}(B)$ does not contain an infinite chain then $\mathrm{T}^{d}(B)$ is well founded and the height function satisfies

$$
\begin{aligned}
& h\left(\emptyset, \mathrm{T}^{d}(B)\right)= \\
& \sup \left\{h\left((a), \mathrm{T}^{d}(B)\right)+1:(a) \in \mathrm{T}(B)\right\}=\sup \left\{h\left(\emptyset, \mathrm{T}^{d}\left({ }_{(a)} B\right)\right)+1:(a) \in \mathrm{T}(B)\right\} .
\end{aligned}
$$

Induction on the height gives then that $\mathrm{T}(B)$ is well ordered under the lexicographic order. The order type of $\mathrm{T}$ being at most $\omega^{\alpha}$ where $\alpha:=h\left(\emptyset, \mathrm{T}^{d}(B)\right)$. From this fact, we deduce:

Lemma 2.3 [20] Every thin block, and in particular every barrier, is well ordered under the lexicographic order.

This allows to associate with every barrier its order-type. We note that $\omega$ is the least possible order-type. An ordinal $\gamma$ is the order-type of a barrier if and only if $\gamma=\omega^{\alpha} \cdot n$ where $n<\omega$ and $n=1$ if $\alpha<\omega$ [1]. Every barrier contains a barrier whose order-type is an indecomposable ordinal.

Definition 2.4 A map $f$ from a barrier $B$ into a poset $P$ is good if there are $s, t \in B$ with $s \triangleleft t$ and $f(s) \leq f(t)$. Otherwise $f$ is bad.

Let $\alpha$ be a denumerable ordinal. A poset $P$ is $\alpha$-better-quasi-ordered if every map $f$ : $B \rightarrow P$, where $B$ is a barrier of order type at most $\alpha$, is good.

$A$ poset $P$ is better-quasi-ordered if it is $\alpha$-better-quasi-ordered for every denumerable ordinal $\alpha$.

It is known and easy to see that a poset $P$ is $\omega$-better-quasi-ordered if and only if it is well-quasi-ordered. Remember that we abbreviate better-quasi-order by bqo. Since every barrier contains a barrier with indecomposable order type, only barriers with indecomposable order type need to be taken into account in the definition of bqo. In particular, we only need to consider $\alpha$-bqo for indecomposable ordinals $\alpha$. If $\alpha<\alpha^{\prime}$ are indecomposable ordinals there exist posets which are $\alpha$-bqo and not $\alpha^{\prime}$-bqo, see [14] .

We will need the following results of Nash-Williams (for proofs in the context of $\alpha$-bqo, see [16] or [22]):

Lemma 2.5 Let $P$ and $Q$ be partial orders, then:

(a) Finite partial orders and well ordered chains are bqo.

(b) If $P, Q$ are $\alpha$-bqo then the direct sum $P \oplus Q$ and the direct product $P \times Q$ are $\alpha$-bqo.

(c) If $P$ is $\alpha$-bqo and $f: P \longrightarrow Q$ is order-preserving then $f(P)$ is $\alpha$-bqo.

(d) If $P$ embeds into $Q$ and $Q$ is $\alpha$-bqo then $P$ is $\alpha$-bqo.

(e) If $C \subseteq \mathfrak{P}(P)$ is $\alpha$-bqo then the set of finite unions of members of $C$ is $\alpha$-bqo. 
(f) If $P$ is $\alpha$-bqo then $\mathfrak{P}_{<\omega}(P)$ is $\alpha$-bqo.

We will use repeatedly the following:

Remarks 2.6 It follows from Item $(e)$ that if $P$ is $\alpha$-bqo then $\mathbf{I}_{<\omega}(P)$ is $\alpha$-bqo which in turn implies that if $P$ is $\alpha$-bqo then $A M(P)$ is $\alpha$-bqo with respect to the domination quasi-order. (Because the order type of a barrier is at least $\omega$ we will always assume that $\alpha \geq \omega$.) If $P$ is $\alpha$-bqo then it is $\omega$-bqo and hence well-quasi-ordered and hence does not contain infinite antichains. Then $A M(P)$ embeds into $A(P)$ which in turn embeds into $\mathbf{I}_{<\omega}(P)$.) It follows from Item $(d)$ that if $P$ is bqo then every restriction of $P$ to a subset of its elements is also bqo.

\subsection{Barrier constructions}

Let $B$ be a subset of $[\mathbb{N}]^{<\omega}$. See Section 7 for notation.

If $B$ is a block then $B^{2}$ is a block and if $B$ is a thin block then $B^{2}$ is a thin block. Moreover, if $B$ is a thin block, and $u \in B^{2}$, then there is a unique pair $s, t \in B$ such that $s \triangleleft t$ and $u=s \cup t$. If $B$ is a block, then $\bigcup_{*} B=\bigcup B \backslash\{\min (\bigcup B)\}$ and ${ }_{*} B$ is a block. Moreover, if $B$ is well ordered under the lexicographic order then ${ }_{*} B$ is well ordered too and if the type of $B$ is an indecomposable ordinal $\omega^{\gamma}$ then the type of ${ }_{*} B$ is at most $\omega^{\gamma}$.

If $C$ is a block and $B:=C^{2}$ then ${ }_{*} B=C \backslash C_{(a)}$, where $a$ is the least element of $\bigcup C$.

The following Lemma is well known and follows easily from the definition.

Lemma 2.7 If $B$ is a barrier, then $B^{2}$ is a barrier and if $B$ has type $\alpha$ then $B^{2}$ has type $\alpha \cdot \omega$.

We recall the following construction due to Marcone [15]. Let $B$ be a subset of $[\mathbb{N}]^{<\omega}$ and let $B^{\circ}$ be the set of all elements $s \in B$ with the property that for all $i \in \bigcup B$ with $i<s(0)$ there is an element $t \in B$ with $(i) \cdot{ }_{*} s \leq_{i n} t$. In other words $s \in B^{\circ}$ if $(i) \cdot{ }_{*} s \in \mathrm{T}(B)$ for all $i \in \bigcup B$ with $i<s(0)$. Let $\check{B}:=\left\{{ }_{*} s: s \in B^{\circ}\right\} \backslash\{\emptyset\}$.

Lemma 2.8 below was given by A.Marcone, see [15] Lemma 8 pp. 343.

Lemma 2.8 Let $B$ be a thin block of type larger than $\omega$, then:

1. $\check{B}$ is a thin block.

2. For every $u \in(\check{B})^{2}$ there is some $s \in B$ such that $s \leq_{i n} u$.

3. If the type of $B$ is at most $\omega^{\gamma}$ then $\check{B}$ contains a barrier of type at most $\omega^{\gamma}$ if $\gamma$ is a limit ordinal and at most $\omega^{\gamma-1}$ otherwise.

Remark 2.9 We may note that if $B$ is a barrier and $u:=s^{\prime} \cup t^{\prime} \in(\check{B})^{2}$, then for every $s \in B$ such that $s \leq_{\text {in }} u$ we have $s^{\prime} \leq_{\text {in }} s$. Indeed, otherwise $s \leq_{\text {in }} s^{\prime}$, but $s^{\prime}:={ }_{*} s^{\prime \prime}$ for some $s^{\prime \prime} \in B$, hence $s \subseteq s^{\prime \prime}$ contradicting the fact that $B$ is a barrier. 
A barrier $B$ is end-closed if

$$
s \leq_{\text {end }} t \quad \text { and } \quad s \in B \quad \text { implies } \quad t \in B .
$$

For example, $[\mathbb{N}]^{n}$ is end-closed for every $n, n \geq 1$, as well as the barrier $B:=\{s \in$ $\mathbb{N}<\omega: l(s)=s(0)+2\}$.

If $B, C$ are two barriers with the same domain, the set $B * C:=\{s \cdot t: s \in C, t \in$ $B, \lambda(s)<t(0)\}$ is a barrier, the product of $B$ and $C$, see [20]. Its order-type is $\omega^{\gamma+\beta}$ if $\omega^{\gamma}$ and $\omega^{\beta}$ are the order-types of $B$ and $C$ respectively. For example, the product $[\bigcup B]^{1} * B$ is end-closed. Provided that $B$ has type $\omega^{\beta}$, it has type $\omega^{1+\beta}$. The converse holds, namely:

Fact 2.10 The set $D \subseteq \mathbb{N}<\omega$ is an end-closed barrier of type larger than $\omega$ if and only if $D_{*}$ is a barrier and $D:=\left[\bigcup D_{*}\right]^{1} * D_{*}$.

Lemma 2.11 Every barrier $B$ contains an end-closed subbarrier $B^{\prime}$.

Proof. Induction on the order-type $\beta$ of $B$.

If $\beta:=\omega$ then $B=[\bigcup B]^{1}$ and we may set $B^{\prime}:=B$.

Suppose $\beta>\omega$ and every barrier of type smaller than $\beta$ contains an end-closed subbarrier.

The set $S(B):=\{i \in \bigcup B:(i) \in B\}$ is an initial segment of $\bigcup B$. (Indeed, let $i \in S(B)$ and $j<i$ with $j \in \bigcup B$. Select $X \in[\bigcup B]^{\omega}$ such that $(j, i) \leq_{i n} X$. Since $B$ is a barrier, $X$ has an initial segment $s \in B$. Since $B$ is an antichain w.r.t. inclusion $i \notin s$, hence $s=(j)$.) The type of $B$ is larger than $\omega$, hence $S(B) \neq \bigcup B$. Set $i_{0}:=\min (\bigcup B \backslash S(B))$.

The set ${ }_{\left(i_{0}\right)} B$ is a barrier because $i_{0} \notin S(B)$. Hence induction applies providing some $X_{0} \subseteq \cup B \backslash\left(S(B) \cup\left\{i_{0}\right\}\right)$ such that $\left\{s:\left(i_{0}\right) \cdot s \in B \cap\left[X_{0}\right]^{<\omega}\right\}$ is an end-closed barrier of domain $X_{0}$. It follows that $\left\{i_{0}\right\} \cup X_{0} \subseteq \cup B$.

Starting with $\left(i_{0}, X_{0}\right)$ we construct a sequence $\left(i_{n}, X_{n}\right)_{n<\omega}$ such that for every $n<\omega$ :

1. $\left\{s:\left(i_{n}\right) \cdot s \in B \cap\left[X_{n}\right]^{<\omega}\right\}$ is an end-closed barrier of domain $X_{n}$.

2. $\left\{i_{n+1}\right\} \cup X_{n+1} \subseteq X_{n}$.

Let $n<\omega$. If $\left(i_{m}, X_{m}\right)_{m<n}$ is defined for all $m<n$ replace $B$ by $B \cap\left[X_{n-1}\right]^{<\omega}$ in the construction of $i_{0}$ and $X_{0}$ to obtain $i_{n}$ and $X_{n}$.

Then for $X:=\left\{i_{n}: n<\omega\right\}$ set $B^{\prime}:=B \cap[X]^{<\omega}$.

\subsection{On the comparison of blocks}

Fact 2.12 Let $B, B^{\prime}$ be two thin blocks. If $B^{\prime} \leq_{i n} B$ then for all $s, t \in B$ and $s^{\prime}, t^{\prime} \in B^{\prime}$ :

(a) $B^{\prime} \subseteq \bigcup B$

(b) If $s \leq_{\text {in }} s^{\prime}$ then $s=s^{\prime}$, hence $\leq_{\text {in }}$ is a partial order on thin blocks.

(c) If $\bigcup B=\bigcup B^{\prime}$ then for every $s \in B$ there is some $s^{\prime} \in B^{\prime}$ such that $s^{\prime} \leq_{\text {in }} s$. 
(d) If $s^{\prime} \triangleleft t^{\prime}$ then $s \triangleleft t$ for some $s, t \in B$ with $s^{\prime} \leq_{\text {in }} s$ and $t^{\prime} \leq_{\text {in }} t$.

(e) The set $B^{\prime \prime}:=B^{\prime} \cup D$ with $D:=\left\{s \in B: \forall s^{\prime} \in B^{\prime}\left(s^{\prime} \not Z_{\text {in }} s\right)\right\}$ is a thin block and $\bigcup B^{\prime \prime}=\bigcup B$ and $B^{\prime \prime} \leq_{\text {in }} B$.

Proof. $(a),(b),(c)$ follow from the definitions.

$(d)$. Let $s^{\prime}, t^{\prime} \in B^{\prime}$ with $s^{\prime} \triangleleft t^{\prime}$ and let $t^{\prime \prime}:=s^{\prime} \cup t^{\prime}$. Since $\bigcup B^{\prime} \subseteq \cup B, t^{\prime \prime} \subseteq \cup B$. Let $X \in[\bigcup B]^{\omega}$ such that $t^{\prime \prime} \leq_{\text {in }} X$. There are $s, t \in B$ such that $s \leq_{\text {in }} X$ and $t \leq_{\text {in } *} X$. We have $s \triangleleft t$. It follows from $(b)$ that $s^{\prime} \leq_{\text {in }} s$ and $t^{\prime} \leq_{\text {in }} t$.

(e) $\bigcup B^{\prime \prime}=\bigcup B^{\prime} \cup \bigcup D$ and $\bigcup B^{\prime} \subseteq \bigcup B$ imply $\bigcup B^{\prime \prime} \subseteq \bigcup B$. For the converse, let $x \in \bigcup B \backslash \bigcup B^{\prime}$. Since $B$ is a block there is some $s \in B$ having $x$ as first element. Clearly $s \in D$, hence $x \in D$, proving $\bigcup B^{\prime \prime}=\bigcup B$. From the definition, $B^{\prime \prime}$ is an antichain. Now, let $X \subseteq B$. We prove that some initial segment $s^{\prime \prime}$ belongs to $B^{\prime \prime}$. Since $B$ is a block, some initial segment $s$ of $X$ belongs to $B$. If $s \in D$ set $s^{\prime \prime}:=s$. Otherwise some initial segment $s^{\prime}$ of $s$ is in $B^{\prime}$. Set $s^{\prime \prime}:=s^{\prime}$.

Let $f: B \rightarrow P$ and $f^{\prime}: B^{\prime} \rightarrow P$ be two maps. Set $f^{\prime} \leq_{\text {in }} f$ if $B^{\prime} \leq_{\text {in }} B$ and $f^{\prime}\left(s^{\prime}\right)=f(s)$ for every $s^{\prime} \in B^{\prime}, s \in B$ with $s^{\prime} \leq_{\text {in }} s$. Let $\mathcal{H}_{X}(P)$ be the set of maps $f: B \rightarrow P$ for which $B$ is a thin block with domain $X$.

Fact 2.13 Let $f: B \rightarrow P$ and $f^{\prime}: B^{\prime} \rightarrow P$ with $f^{\prime} \leq_{\text {in }} f$. If $B^{\prime}$ and $B$ are thin blocks then $B^{\prime}$ extends to a thin block $B^{\prime \prime}$ and $f^{\prime}$ to a map $f^{\prime \prime}$ such that $\bigcup B^{\prime \prime}=\bigcup B$ and $f^{\prime \prime} \leq_{\text {in }} f$.

Proof. Applying $(e)$ of Fact 2.12 , set $B^{\prime \prime}:=B^{\prime} \cup D$ and define $f^{\prime \prime}$ by setting $f^{\prime \prime}\left(s^{\prime \prime}\right):=f(s)$ if $s^{\prime \prime} \in D$ and $f^{\prime \prime}\left(s^{\prime \prime}\right):=f^{\prime}\left(s^{\prime \prime}\right)$ if $s^{\prime \prime} \in B^{\prime \prime}$. Then $f^{\prime \prime} \leq_{\text {in }} f$.

Fact 2.14 Let $P$ be a poset and $X \in \mathbb{N}^{\omega}$, then:

(a) The relation $\leq_{i n}$ is an order on the collection of maps $f$ whose domain is a thin block and whose range is $P$.

(b) Every $\leq_{i n}$-chain has an infimum on the set $\mathcal{H}_{X}(P)$.

(c) An element $f$ is minimal in $\mathcal{H}_{X}(P)$ if and only if every $f^{\prime}$ with $f^{\prime} \leq_{\text {in }} f$ is the restriction of $f$ to a sub-block of the domain of $f$.

(d) If $f$ is minimal in $\mathcal{H}_{X}(P)$ and $f^{\prime} \leq_{\text {in }} f$ has domain $C$ then $f^{\prime}$ is minimal in $\mathcal{H}_{\cup C}(P)$.

(e) Let $B$ be a thin block and $f: B \rightarrow P$. If $f$ is bad and $f^{\prime} \leq_{\text {in }} f$ then $f^{\prime}$ is bad.

Proof. (a) Obvious.

(b) Let $\mathcal{D}:=\left\{f_{\alpha}: B_{\alpha} \rightarrow P\right\}$ be a $\leq_{i n}$-chain of maps. Let $\mathcal{C}:=\{\operatorname{dom}(f): f \in \mathcal{D}\}$. Then $D:=\left\{s \in \bigcup \mathcal{C}: \forall s^{\prime} \in \bigcup \mathcal{C}\left(s^{\prime} \nless s\right)\right\}$ is a thin block and the infimum of $\mathcal{C}$. For $s \in D$, let $f^{\prime}(s)$ be the common value of all maps $f_{\alpha}$. This map is the infimum of $\mathcal{D}$.

(c) Apply Fact 2.13.

(d) Follows from $(c)$.

(e) Apply $(d)$ of Fact 2.12. 
Lemma 2.15 Let $f$ be a map from a thin block $B$ into $P$ and let $\mathcal{F}:=\left\{f^{\prime} \in \mathcal{H}_{\cup B}(P)\right.$ : $\left.f^{\prime} \leq_{\text {in }} f\right\}$. Then there is a minimal $f^{\prime} \in \mathcal{F}$ such that $f^{\prime} \leq_{\text {in }} f$.

Proof. Follows from Fact $2.14(b)$ using Zorn's Lemma.

Lemma 2.16 Let $f: B \rightarrow P$ a bad map. If $P$ is wqo and $f$ is minimal then there is an end-closed barrier $B^{\prime} \subseteq B$ such that:

$$
s<_{\text {end }} t \text { in } B^{\prime} \Rightarrow f(s)<f(t) \text { in } P \text {. }
$$

Proof. Let $B_{1}$ be a an end-closed subbarrier of $B$ and let $C_{1}:=\left\{s \cdot(b): s \in B_{1}, b \in\right.$ $\left.\bigcup B_{1}, b>\lambda(s)\right\}=\left[\bigcup B_{1}\right]^{1} * B_{1}$. Divide $C_{1}$ into three parts $D_{i}, i<3$, with $D_{i}:=\left\{s^{\prime} \cdot(a b) \in\right.$ $\left.C_{1}: f\left(s^{\prime} \cdot(a)\right) \rho_{i} f\left(s^{\prime} \cdot(b)\right)\right\}$ where $\rho_{0}$ is the equality relation, $\rho_{1}$ is the strict order $<$ and $\rho_{2}$ is $\not \leq$ the negation of the order relation on $P$.

Since $C_{1}$ is a barrier, Nash-Williams 's partition theorem (Theorem $2.2(b)$ ) asserts that one of these parts contains a barrier $D$. Let $X$ be an infinite subset of $\bigcup C_{1}$ such that $D=C_{1} \cap[X]^{<\omega}$.

The inclusion $D \subseteq D_{2}$ is impossible. Otherwise, let $s \in B_{1}$ such that $s \leq_{\text {in }} X$, set $Y:=X \backslash s_{*}$ and set $g(a):=f\left(s_{*} \cdot(a)\right)$ for $a \in Y$. Then $g$ is a bad map from $Y$ into $P$. This contradicts the fact that $P$ is wqo.

The inclusion $D \subseteq D_{0}$ is also impossible. Otherwise, set $B^{\prime}:=\left\{s^{\prime}: s^{\prime} \cdot(a) \in B_{1} \cap[X]^{<\omega}\right.$ for some $a\}$. For $s^{\prime} \in B^{\prime}$, set $f^{\prime}\left(s^{\prime}\right):=f\left(s^{\prime} \cdot(a)\right)$ where $a \in X$. Since $D \subseteq D_{0}$ the function value $f^{\prime}\left(s^{\prime}\right)$ is well-defined. Since $P$ is wqo and $f$ is bad, the order type of $B_{1}$ is at least $\omega^{2}$, hence $B^{\prime}$ is a barrier. The map $f^{\prime}$ satisfies $f^{\prime} \leq_{i n} f$. According to Fact $2.14(c)$, the minimality of $f$ implies that $f^{\prime}$ is the restriction of $f$ to $B^{\prime}$. Since $B^{\prime}$ is not included into $B$ this is it not the case. A contradiction.

Thus we have $D \subseteq D_{1}$. Set $B^{\prime}:=B_{1} \cap[X]^{<\omega}$. Then (2) holds.

\subsection{An application to $S_{\omega}(P)$}

We deduce Theorem 1.1 from the equivalence $(i) \Longleftrightarrow(i i)$ in the following result. Without clause (ii), the result is due to A.Marcone [15]. Without Marcone's result our proof only shows that under clause (ii) $P$ is $\alpha$-bqo. This suffices to prove Theorem 1.1 but the result below is more precise.

Theorem 2.17 Let $\alpha$ be a denumerable indecomposable ordinal and $P$ be a poset. Then the following properties are equivalent:

(i) $P$ is $\alpha \omega-b q o$;

(ii) $P$ is $\omega$-bqo and $S_{\omega}(P)$ is $\alpha$-bqo.

(iii) $\mathfrak{P}_{\leq \omega}(P)$ is $\alpha$-bqo

(iv) $\mathfrak{P}(P)$ is $\alpha$-bqo 
Proof. $(i) \Rightarrow(i v)$. Let $B$ be a barrier with order type at most $\alpha$ and $f: B \rightarrow \mathfrak{P}(P)$. If $f$ is bad, let $f^{\prime}: B^{\prime} \rightarrow P$ where $B^{\prime}:=B^{2}$ and $f^{\prime}(s \cup t) \in f(s) \backslash \downarrow f(t)$. (See Equation 6.) This map $f^{\prime}$ is bad and the order type of $B^{\prime}$ is at most $\alpha \omega$.

$($ iv $) \Rightarrow($ iii $)$ Trivial.

(iii) $\Rightarrow$ (ii) $P$ and $S_{\omega}(P)$ identify to subsets of $\mathfrak{P}_{\leq \omega}(P)$, hence are $\alpha$-bqo.

(ii) $\Rightarrow($ i) Suppose that $P$ is not $\alpha \omega$-bqo. Let $\beta$ be the smallest ordinal such that $P$ is not $\beta$-bqo. Then $\beta \leq \alpha \omega$ and $\beta$ is indecomposable.

Case 1. $\beta=\alpha^{\prime} \omega$. According to Marcone [15] the implication (iii) $\Rightarrow(i)$ holds for all denumerable ordinals, hence there is a bad map $f^{\prime}: B^{\prime} \rightarrow \mathfrak{P}_{\leq \omega}(P)$ for which $B^{\prime}$ is a barrier of type at most $\alpha^{\prime}$. (Note $\alpha^{\prime}<\beta$.) Let $X \in \mathfrak{P}_{\leq \omega}(P)$. Since $P$ is wqo, $\downarrow X$ is a finite union of ideals according to Fact 7.1.

Hence there are a finite antichain $A_{X}$ and a finite set $B_{X}$ of strictly increasing sequences such that $\downarrow X=\downarrow A_{X} \cup \downarrow B_{X}$. Let $g: B^{\prime} \rightarrow \mathfrak{P}_{<\omega}(P) \times \mathfrak{P}_{<\omega}\left(S_{\omega}(P)\right)$ defined by $g\left(s^{\prime}\right)=$ $\left(A_{f^{\prime}\left(s^{\prime}\right)}, B_{f\left(s^{\prime}\right)}^{\prime}\right)$. This map is bad. Hence, from $(b)$ and $(f)$ of Lemma 2.5 there is a bad map from a subbarrier $B^{\prime \prime}$ of $B^{\prime}$ into $P$ or into $S_{\omega}(P)$. The latter case is impossible since $S_{\omega}(P)$ is $\alpha$-bqo and so is the former case because of the minimality of $\beta$.

Case 2. Case 1 does not hold, that is $\beta=\omega^{\gamma}$ where $\gamma$ is a limit ordinal it follows that $\beta \leq \alpha$. Let $f: B \rightarrow P$ be a bad map where $B$ is a barrier of type $\beta$.

According to Lemma 2.15 there is a minimal $f^{\prime}: B^{\prime} \rightarrow P$ with $\bigcup B^{\prime}=\bigcup B$ and $f^{\prime} \leq_{\text {in }} f$ and according to Fact $2.14(e)$ the map $f^{\prime}$ is bad. Since $P$ is wqo, Lemma 2.16 applies. Thus $B^{\prime}$ contains a subbarrier $B^{\prime \prime}$ on which $s \leq_{i n} t$ implies $f^{\prime}(s)<f^{\prime}(t)$.

Let $F: B_{*}^{\prime \prime} \rightarrow \mathfrak{P}(P)$ be given by $F\left(s^{\prime}\right):=\downarrow\left\{f^{\prime}(t) \in P: t \in B^{\prime \prime}\right.$ and $\left.s^{\prime} \leq_{\text {in }} t\right\}$.

Claim $1 F\left(s^{\prime}\right)$ is a finite union of non-principal ideals of $P$. Since $P$ is wqo, every initial segment is a finite union of ideals. Hence in order to show that $F\left(s^{\prime}\right)$ is a finite union of non-principal ideals it suffices to show that it contains no maximal element. Let $x \in F\left(s^{\prime}\right)$. Let $t \in B^{\prime \prime}$ such that $s^{\prime} \leq_{\text {in }} t, f^{\prime}(t) \geq x$. Let $u \in B^{\prime \prime}$ such that $t<_{\text {end }} u$. Then $s^{\prime} \leq_{i n} u$ hence $f^{\prime}(u) \in F\left(s^{\prime}\right)$. From Lemma $2.16 f^{\prime}(t)<f(u)$, proving our claim.

Claim $2 F$ is good. Indeed, since $S_{\omega}(P)$ is $\alpha$-bqo, it follows from (e) of Lemma 2.5 that the collection of finite unions of its members is $\alpha$-bqo.

Hence $f^{\prime}$ is good. Indeed, since $F$ is good, there are $s^{\prime}, t^{\prime} \in B_{*}^{\prime \prime}$ such that $s^{\prime} \triangleleft t^{\prime}$ and $F\left(s^{\prime}\right) \subseteq F\left(t^{\prime}\right)$. Let $a:=t^{\prime}\left(l\left(s^{\prime}\right)-1\right)$ then $s^{\prime} \leq_{\text {in }} s:=s^{\prime} \cdot(a) \in B^{\prime \prime}$ then $f^{\prime}(s) \in F\left(s^{\prime}\right)$. Since $F\left(s^{\prime}\right) \subseteq F\left(t^{\prime}\right)$ there is some $t \in B^{\prime \prime}$ such that $t^{\prime} \leq_{\text {in }} t$ and $f^{\prime}(s) \leq f^{\prime}(t)$. Because $s \triangleleft t$ the map $f^{\prime}$ is good.

This contradicts the hypothesis that $f^{\prime}$ is bad and finishes the proof of the theorem.

\section{The set of ideals of a well-quasi-ordered-poset}

In this section, we illustrate the relevance of the notion of ideal w.r.t. well-quasi-ordering.

The usual topology on the power-set $\mathfrak{P}(P)$ is obtained by identifying each subset of $P$ with its characteristic function and giving the resulting space $\{0,1\}^{P}$ the product topology. Endowed with this topology $\mathfrak{P}(P)$ is also called the Cantor space. A basis of open sets of the Cantor space consists of subsets of the form $O(F, G):=\{X \in \mathfrak{P}(P)$ : 
$F \subseteq X$ and $G \cap X=\emptyset$, where $F, G$ are finite subsets of $P$.

The topological closure of $\operatorname{down}(P)$ in $\mathfrak{P}(P)$ is a Stone space which is homeomorphic to the Stone space of Tailalg $(P)$, the Boolean algebra generated by up $(P)$. With the order of inclusion added the closure of $\operatorname{down}(P), \overline{\operatorname{down}(P)}$, is isomorphic to the Priestley space of Taillat $(P)[3]$.

Note that $\mathbf{I}(P)$ is a closed subspace of the closed Cantor space $\mathfrak{P}(P)$. In fact $\mathbf{I}(P)$ is an algebraic lattice, see [9], whose set of (algebraically) compact elements is $\mathbf{I}_{<\omega}(P)$. It follows that $\mathcal{J}\left(\mathbf{I}_{<\omega}(P)\right) \cong \mathbf{I}(P)$. We also note that $\mathcal{J}(P)$ is the set of join-irreducible elements of $\mathbf{I}(P)$.

We have the following properties. We prove only Proposition 3.5, see [3] for the other properties.

Lemma $3.1 \emptyset \notin \overline{\operatorname{down}(P)} \Longleftrightarrow P \in \mathbf{F}_{<\omega}(P)$.

Lemma 3.2 down $(P) \subseteq \mathcal{J}(P) \subseteq \overline{\operatorname{down}(P)} \backslash\{\emptyset\}$. In particular, the topological closures in $\mathfrak{P}(P)$ of down $(P)$ and $\mathcal{J}(P)$ are the same.

A poset $P$ is up-closed if every intersection of two members of $u p(P)$ is a finite union (possibly empty) of members of $u p(P)$.

Proposition 3.3 The following properties for a poset $P$ are equivalent:

(a) $\mathcal{J}(P) \cup\{\emptyset\}$ is closed in the Cantor space $\mathfrak{P}(P)$;

(b) $\mathcal{J}(P)=\overline{\operatorname{down}(P)} \backslash\{\emptyset\}$;

(c) $P$ is up-closed;

(d) $\mathbf{F}_{<\omega}(P)$ is a meet-semi-lattice;

(e) Taillat $(P)=\mathbf{F}_{<\omega}(P) \cup\{P\}$.

Corollary 3.4 The following properties for a poset $P$ are equivalent:

1. $\mathcal{J}(P)$ is closed in $\mathfrak{P}(P)$;

2. $P \in \mathbf{F}_{<\omega}(P)$ and $P$ is up-closed.

Let us recall that a topological space $X$ is scattered if every non-empty subset $Y$ of $X$ contains an isolated point with respect to the topology induced on $Y$. We have:

Proposition 3.5 Let $P$ be a poset. If $P$ is well-quasi-ordered then $\mathcal{J}(P)$ is a compact scattered space whose set of isolated points coincides with down $(P)$. 


\section{Proof.}

Claim $1 \mathcal{J}(P)=\overline{\operatorname{down}(P)}$. Indeed, from $2 \Rightarrow 1$ of Corollary 3.4, $\mathcal{J}(P)$ is closed. Apply the conclusion of Lemma 3.2.

Claim 2 As a subspace of the Cantor space $\mathfrak{P}(P), \mathbf{I}(P)$ is compact and scattered.

As already mentioned $\mathbf{I}(P)$ is closed. To see that it is scattered, let $X$ be a non-empty subset of $\mathbf{I}(P)$. Since $P$ is wqo, $\mathbf{I}(P)$ is well-founded. Select a minimal element $I$ in $X$. Let $G:=\min (P \backslash I)$. Since $P$ is wqo, $G$ is finite, hence $O(\emptyset, G)\left(=\left\{I^{\prime} \in \mathbf{I}(P): G \cap I^{\prime}=\emptyset\right\}\right)$ is a clopen subset of $\mathfrak{P}(P)$. Since $O(\emptyset, G) \cap X=\{I\}, I$ is isolated in $X$.

Claim 3 Let $J \in \mathcal{J}(P)$, then $J$ is isolated in $\mathcal{J}(P)$ if and only $J$ is principal.

Suppose that $J$ is isolated. Then there is a clopen set of the form $O(F, G)$ such that $O(F, G) \cap \mathcal{J}(P)=\{J\}$. Since $J$ is up-directed, there is some $z$ in $J$ which majorizes $F$. Clearly, $\downarrow z \in O(F, G) \cap \mathcal{J}(P)$, hence $J=\downarrow z$, proving that $J$ is principal. Conversely, let $z \in P$. Let $G:=\min (P \backslash \downarrow z)$. Since $P$ is wqo, $G$ is finite. Hence $O(\{z\}, G)$ is a clopen set. This clopen set contains only $\downarrow z$, proving that $\downarrow z$ is isolated in $\mathcal{J}(P)$.

From this result, $\mathcal{J}^{\neg \downarrow}:=\mathcal{J}(P) \backslash \operatorname{down}(P)$, the set of non-principal ideals of $P$, coincides with $\mathcal{J}^{1}(P)$, the first Cantor-Bendixson derivative of $\mathcal{J}(P)$. Our main result establishes a link between the bqo characters of $\mathcal{J}(P)$ and $\mathcal{J}^{1}(P)$. This suggests to look at the other derivatives.

\section{Minimal type posets}

Infinite well-quasi-ordered posets $P$ which are up-directed and whose all ideals distinct from $P$ are non-principal are quite interesting. We say that they have minimal type. They can be characterized in various ways:

Proposition 4.1 Let $P$ be an infinite poset. Then, the following properties are equivalent:

(i) $P$ is wqo and all ideals distinct from $P$ are principal;

(ii) $P$ has no infinite antichain and all ideals distinct from $P$ are finite;

(iii) Every proper initial segment of $P$ is finite.

(iv) Every linear extension of $P$ has order type $\omega$.

(v) $P$ is level-finite, of height $\omega$, and for each $n<\omega$ there is $m<\omega$ such that each element of height at most $n$ is below every element of height at least $m$.

(vi) $P$ embeds none of the following posets: an infinite antichain; a chain of order type $\omega^{\text {dual }}$; a chain of order type $\omega+1$; the direct sum $\omega \oplus 1$ of a chain of order type $\omega$ and a one element chain.

The equivalence between item (iii), (iv) and (v) was given in [21]. One proves

$$
(i) \Rightarrow(i i) \Rightarrow(i i i) \Rightarrow(i v) \Rightarrow(v) \Rightarrow(v i) \Rightarrow(i)
$$

using straightforward arguments.

An easy way of obtaining posets with minimal type is given by the following corollary

THE ELECTRONIC JOURNAL OF COMBINATORICS 13 (2006), \#R101 
Corollary 4.2 Let $n$ be an integer and $P$ be a poset. The order on $P$ is the intersection of $n$ linear orders of order type $\omega$ if and only if $P$ is the intersection of $n$ linear orders and $P$ has minimal type.

Minimal type posets occur quite naturally in symbolic dynamic. Indeed, let $S: A^{\omega} \rightarrow$ $A^{\omega}$ be the shift operator on the set $A^{\omega}$ of infinite sequences $s:=\left(s_{n}\right)_{n<\omega}$ of members of a finite set $A$ (that is $S(s):=\left(s_{n+1}\right)_{n<\omega}$ ). A subset $F$ of $A^{\omega}$ is invariant if $S(F) \subseteq F$. As it is well-known, every compact (non-empty) invariant subset contains a minimal one. To a compact invariant subset $F$ we may associate the set $\mathcal{A}(F)$ of finite sequences $s:=\left(s_{0}, \ldots, s_{n-1}\right)$ such that $s$ is an initial segment of some member of $F$. Looking as these sequences as words, we may order $\mathcal{A}(F)$ by the factor ordering: a sequence $s$ being a factor of a sequence $t$ if $s$ can be obtained from $t$ by deleting an initial segment and an end segment of $t$.

We have then

Theorem 4.3 $\mathcal{A}(F)$ has minimal type if and only if $F$ is a minimal compact invariant subset.

\section{Maximal antichains, "pred" and "succ"}

Let $P$ be a poset. We consider both $A(P)$ and $A M(P)$ to be ordered by domination. The main result of this section will be that if $A M(P)$ is $\alpha$-bqo then $P$ is $\alpha$-bqo.

Our first aim is to prove that if $A M(P)$ is well founded then $P$ is well founded. To this end we will associate with every element $x \in P$ an antichain $\varphi(x)$ and investigate the connection between $x$ and $\varphi(x)$. First the following:

Lemma 5.1 Let $P$ be a poset and $X \subseteq A(P)$. Then the following properties are equivalent:

(i) $X$ is the minimum of $A M(P)$.

(ii) $X$ is a minimal element of $A M(P)$.

(iii) $P=\uparrow X$.

Proof. Implications $(i i i) \Rightarrow(i) \Rightarrow(i i)$ are obvious.

(ii) $\Rightarrow$ (iii): Suppose for a contradiction that $P \backslash \uparrow X \neq \emptyset$. For every element $z \in P \backslash \uparrow X$ there is an element $x \in X$ with $z<x$. Let $Y$ be a maximal antichain of the set $P \backslash \uparrow X$ and $Z=X \backslash \uparrow Y$. Then $Z \cup Y$ is a maximal antichain which is strictly dominated by $X$.

Let $P$ be a poset. For $x \in P$ let $\Phi(x):=\{z \in P: z \nless x\}$ and let $\varphi(x):=\min \Phi(x)$. Note that $x \in \varphi(x)$.

Lemma 5.2 1. $A M(\Phi(x)) \subseteq A M(P)$. 


$$
\text { 2. } x<y \Longleftrightarrow \varphi(x)<\varphi(y) \text { and } x \notin \varphi(y) \text { for all } x, y \in P \text {. }
$$

Proof. 1. Let $A \in A M(\Phi(x))$. Since $x$ is minimal in $\Phi(x)$ there exists $y \in A$ with $x \leq y$. Hence if $z \notin \Phi(x)$ we have $z<y$ and this shows $A \in A M(P)$.

2. Suppose $x<y$, then $\Phi(y) \subset \Phi(x)$. Hence $\varphi(y):=\min \Phi(y) \leq \min \Phi(x)=: \varphi(x)$. Then $\varphi(x)<\varphi(y)$ because $\varphi(x) \ni x \notin \varphi(y)$.

Conversely, suppose $\varphi(x)<\varphi(y)$ and $x \notin \varphi(y)$. If $x \nless y$, then the definition of $\varphi(y)$ insures that $x^{\prime} \leq x$ for some $x^{\prime} \in \varphi(y)$. Since $\varphi(x)<\varphi(y)$, we have $x=x^{\prime}$ proving $x \in \varphi(y)$, a contradiction.

Note that if $\varphi(x)$ is a maximal antichain for every $x \in P$ and $A M(P)$ is well founded then we obtain, using Lemma 5.2, that $P$ is well founded. Actually we will show below that if $A M(P)$ is well-founded then $\varphi(x)$ is a maximal antichain.

Let $x \in P$ and $\mathcal{F} \subseteq \mathfrak{P}(P)$. Then define $\mathcal{F}(x):=\{F \in \mathcal{F}: x \in F\}$ and $\operatorname{Inc}_{P}(x):=$ $\{y \in P: x$ and $y$ are incomparable $\}$. Note that $A M\left(\operatorname{Inc}_{P}(x)\right) \cong A M(P)(x)$. $(\cong$ is the order isomorphism between he maximal antichains of $\operatorname{Inc}_{P}(x)$ and the maximal antichains of $A M(P)(x)$ both ordered under domination.) According to Item 1 of Lemma $5.2 A M(\Phi(x)) \subseteq A M(P)$. Hence, if $A M(P)$ is well-founded then $A M(\Phi(x))$ is wellfounded. Let $S$ be a minimal element of $A M(\Phi(x))$. It follows then from Lemma 5.1 that $S$ is the minimum of $A M(\Phi(x))$ and $\uparrow S=\Phi(x)$. Hence $S=\varphi(x)$. That is: $\varphi(x)$ is the least maximal antichain of $P$ containing $x$.

Also, if $P$ is well-founded then $\Phi(x)$ is well-founded, hence $\uparrow \varphi(x)=\Phi(x)$ which in turned implies, using Lemma 5.1, that $\varphi(x)$ is the least maximal antichain of $P$ containing $x$. Hence we established the following Lemma:

Lemma 5.3 If $A M(P)$ is well-founded or if $P$ is well founded then for all $x, y \in P$ :

1. $\uparrow \varphi(x)=\Phi(x)$.

2. $\varphi(x)$ is the minimum of all maximal antichains of $P$ containing $x$.

3. $x<y \Longleftrightarrow \varphi(x)<\varphi(y)$ and $x \notin \varphi(y)$. (Item 2. of Lemma 5.2).

4. $P$ is well founded.

Associated with the quasi order $\left(P ; \leq_{\text {pred }}\right)$ is the equivalence relation $\equiv$ equal to the set $\left\{(x, y): x \leq_{\text {pred }} y\right.$ and $\left.y \leq_{\text {pred }} x\right\}$. Let $\left(P ; \leq_{\text {pred }}\right) / \equiv$ be the quotient equipped with the order induced by $\leq_{\text {pred }}$. Let $\pi$ be the canonical map of $\left(P ; \leq_{\text {pred }}\right)$ to $\left(P ; \leq_{\text {pred }}\right) / \equiv$. For every subset $S$ of $P$ let $\bar{\pi}(S):=\{\pi(s): s \in \downarrow S\}$. That is $\bar{\pi}(S)$ is the least initial segment of $\left(P ; \leq_{\text {pred }}\right) / \equiv$ containing the image of $S$ under $\pi$.

Theorem 5.4 Let $P$ be a poset and $Q:=\left(P ; \leq_{\text {pred }}\right) / \equiv$.

1. The function $\bar{\pi}$ induces an embedding of $A M(P)$ into $\mathbf{I}(Q)$; if moreover $P$ has no infinite antichain then the image of $A M(P)$ is a subset of $\mathbf{I}_{<\omega}(Q)$.

2. If $P$ is well founded then $Q$ embeds into $A M(P)$. 
3. $\bar{\pi}$ induces an embedding of $\mathcal{J}^{\neg}(P)$ into $\mathcal{J}^{\neg}(Q)$.

4. If $P$ has no infinite antichain then this embedding is surjective, hence $\mathcal{J}^{\neg}(P) \cong$ $\mathcal{J}^{\neg \downarrow}(Q)$.

\section{Proof.}

Item 1: By definition, $\bar{\pi}(S) \in \mathbf{I}(Q)$ for every $S \in \mathfrak{P}(P)$. Hence $\bar{\pi}$ induces a map from $A M(P)$ into $\mathbf{I}(Q)$. Let $A, B \in A M(P)$ with $\bar{\pi}(A) \subseteq \bar{\pi}(B)$. For every $a \in A$ there is an element $b \in B$ so that $a$ and $b$ are related under $\leq$. Assume for a contradiction that $b<a$. Then $\pi(b)<\pi(a)$. Because $\bar{\pi}(A) \subseteq \bar{\pi}(B)$ there is a $c \in B$ with $\pi(a) \leq \pi(c)$. This implies $a \leq_{\text {pred }} c$ thus $b<c$ a contradiction. Hence $\bar{\pi}(A) \subseteq \bar{\pi}(B)$ implies that $A$ is less than or equal to $B$ in the domination order which in turn implies that if $\bar{\pi}(A)=\bar{\pi}(B)$ then $A=B$. If $A$ is less than or equal to $B$ in the domination order then $\bar{\pi}(A) \subseteq \bar{\pi}(B)$ and hence we conclude that $\bar{\pi}$ is an embedding of $A M(P)$ into $\mathbf{I}(Q)$.

Item 2: According to Lemma $5.3 \varphi(x) \in A M(P)$ for every $x \in P$. Moreover, the map $\varphi$ induces an embedding from $Q$ into $A M(P)$.

Item 3: Let $J \in \mathcal{J}^{\urcorner} \downarrow(P)$. By definition of $\bar{\pi}, \bar{\pi}(J) \in \mathbf{I}(Q)$. Since $\pi$ is increasing with respect to the $\leq$ order on $P$ we have $\pi(J) \in \mathcal{J}(Q)$. Suppose that $\pi(J)$ has a largest element, say $x$. There is $y \in J$ such that $\pi(y)=x$. Since $J \in \mathcal{J}^{\neg}(P)$ there is some $z \in J$ with $y<z$. Hence $x=\pi(y)<\pi(z) \in \bar{\pi}(J)$. A contradiction.

Let $J, J^{\prime} \in \mathcal{J}^{\neg}(P)$. If $J \subseteq J^{\prime}$ then clearly $\bar{\pi}(J) \subseteq \bar{\pi}\left(J^{\prime}\right)$. Suppose $J \nsubseteq J^{\prime}$. Let $x \in J \backslash J^{\prime}$. Since $J$ is not principal, there is some $x^{\prime} \in J$ such that $x<x^{\prime}$. Since $J^{\prime}$ is an initial segment, $x^{\prime} \notin J^{\prime}$. Assume for a contradiction that $\pi\left(x^{\prime}\right) \in \bar{\pi}\left(J^{\prime}\right)$. Then there is an $x^{\prime \prime} \in J^{\prime}$ such that $\pi\left(x^{\prime}\right) \leq \pi\left(x^{\prime \prime}\right)$ hence $x^{\prime} \leq_{\text {pred }} x^{\prime \prime}$. Therefore $x<x^{\prime \prime}$ follows from $x<x^{\prime}$. Since $J^{\prime}$ is an initial segment, we have $x \in J^{\prime}$ contradicting the choice of $x$.

This proves that $\bar{\pi}(J) \nsubseteq \mathbb{\pi}\left(J^{\prime}\right)$. Hence $\bar{\pi}$ is an embedding.

Item 4: Let $K \in \mathcal{J}^{\urcorner}(Q)$. Let $J:=\{x \in P: \pi(x) \in K\}$. The set $J$ is an initial segment of $P$ since $\pi$ is order preserving. The set $J$ is a finite union of ideals since $P$ has no infinite antichain; see Fact 7.1. Let $J:=J_{1} \cup \cdots \cup J_{k}$. We have $K=\bar{\pi}(J)=\bar{\pi}\left(J_{1}\right) \cup \cdots \cup \bar{\pi}\left(J_{k}\right)$. From the fact that $K$ is an ideal it follows that $K=\bar{\pi}\left(J_{i}\right)$ for some $i$. Since $K$ is not principal, $J_{i}$ cannot be principal.

We derive Theorem 1.7 from the following result:

Theorem 5.5 Let $P$ be a poset with no infinite antichain and $\alpha$ be a countable ordinal. The following properties are equivalent:

(i) $P$ is $\alpha$-bqo.

(ii) $\left(P ; \leq_{\text {succ }}\right)$ is $\alpha$-bqo.

(iii) $\left(P ; \leq_{\text {pred }}\right)$ is $\alpha$-bqo.

(iv) $\left(P ; \leq_{\text {crit }}\right)$ is $\alpha$-bqo.

(v) $A M(P)$ is $\alpha$-bqo. 
Proof. Implications $(i) \Longrightarrow(i v) \Longrightarrow$ (iii) follow from the sequence of inclusions ( $\leq$ )$\subseteq\left(\leq_{\text {crit }}\right) \subseteq\left(\leq_{\text {pred }}\right)$ and the implication $(i v) \Longrightarrow(i i)$ follows from the inclusion $\left(\leq_{\text {crit }}\right) \subseteq\left(\leq_{\text {succ }}\right)$.

(iii) $\Longleftrightarrow(v)$ Suppose $A M(P)$ is $\alpha$-bqo then $P$ is well-founded according to Lemma 5.3 and hence according to item 2 of Theorem $5.4,\left(P ; \leq_{\text {pred }}\right) / \equiv$ embeds into $A M(P)$ implying that $\left(P ; \leq_{\text {pred }}\right)$ is $\alpha$-bqo. Conversely, suppose $\left(P ; \leq_{\text {pred }}\right)$ is $\alpha$-bqo. Then $\mathbf{I}_{<\omega}\left(\left(P ; \leq_{\text {pred }}\right) / \equiv\right.$ ) is $\alpha$-bqo according to item e of Lemma 2.5. From item 1 of Theorem 5.4, the poset $A M(P)$ embeds into $\mathbf{I}_{<\omega}\left(\left(P ; \leq_{\text {pred }}\right) / \equiv\right)$ and hence is $\alpha$-bqo.

We prove implications $(i i) \Longrightarrow(i)$ and $(i i i) \Longrightarrow(i)$.

Let $Q$ be equal to $\left(P ; \leq_{\text {succ }}\right)$ or equal to $\left(P ; \leq_{\text {pred }}\right)$. Suppose that $Q$ is $\alpha$-bqo. Since $(\leq) \subseteq\left(\leq_{\text {succ }} \cap \leq_{\text {pred }}\right)$, the partial order $P$ is well-founded and since it has no infinite antichain it is wqo. If $P$ is not $\alpha$-bqo there is a barrier $B$ of type at most $\alpha$ and a bad map $f: B \rightarrow P$. From Lemma 2.15 there is a minimal $f^{\prime}: B^{\prime} \rightarrow P$ such that $\bigcup B^{\prime}=\bigcup B$ and $f^{\prime} \leq_{i n} f$. According to Fact $2.14(e)$ the map $f^{\prime}$ is bad. Since $P$ is wqo, Lemma 2.16 applies. Thus $B^{\prime}$ contains an end-closed subbarrier $B^{\prime \prime}$ on which

$$
s<_{\text {end }} t \Longrightarrow f^{\prime}(s)<f^{\prime}(t) .
$$

Suppose $Q:=\left(P ; \leq_{\text {succ }}\right)$. Since $\left(P ; \leq_{\text {succ }}\right)$ is $\alpha$-bqo, the function $f^{\prime}$ cannot be bad thus there are $s, t \in B^{\prime \prime}$ such that $s \triangleleft t$ and $f^{\prime}(s) \leq_{\text {succ }} f^{\prime}(t)$. Pick $t^{\prime} \in B^{\prime \prime}$ such that $t<_{\text {end }} t^{\prime}$. From (3) we have $f^{\prime}(t)<f^{\prime}\left(t^{\prime}\right)$. According to the definition of $\left(P ; \leq_{\text {succ }}\right)$, we have $f^{\prime}(s) \leq f^{\prime}\left(t^{\prime}\right)$. Since $s \triangleleft t^{\prime}$ it follows that $f^{\prime}$ is good for $P$. A contradiction.

Suppose $Q:=\left(P ; \leq_{\text {pred }}\right)$. For $s \in B^{\prime \prime}$ set $s^{+}:=s_{*} \cdot(a)$ where $a$ is the successor of $\lambda(s)$ in $\bigcup B^{\prime \prime}$. Set $f^{\prime+}(s):=f^{\prime}\left(s^{+}\right)$. Since $\left(P ; \leq_{\text {pred }}\right)$ is $\alpha$-bqo there are $s$ and $t$ such that $s \triangleleft t$ and $f^{\prime+}(s) \leq_{\text {pred }} f^{\prime+}(t)$, that is $f^{\prime}\left(s^{+}\right) \leq_{\text {pred }} f^{\prime}\left(t^{+}\right)$. Since $s<_{\text {end }} s^{+}$we have $f^{\prime}(s)<f^{\prime}\left(s^{+}\right)$. According to the definition of $\left(P ; \leq_{\text {pred }}\right)$, this gives $f^{\prime}(s)<f^{\prime}\left(t^{+}\right)$. Since $s \triangleleft t^{+}$, the function $f^{\prime}$ cannot be bad. A contradiction.

\section{Maximal antichains with a prescribed size}

\subsection{Two element maximal antichains}

Definition 6.1 Let $P$ be a poset. The structure $(P(2) ; \leq)$ is defined on $P(2):=P \times 2$ so that:

$$
(x, i) \leq(y, j) \text { if } \begin{cases}i=j & \text { and } x \leq y, \text { or } \\ i=0 \text { and } j=1 & \text { and there exist incomparable elements } \\ & x^{\prime}, y^{\prime} \in P \text { with } x \leq x^{\prime} \text { and } y^{\prime} \leq y .\end{cases}
$$

It is easy to see that $P(2)$ is a poset.

Lemma 6.2 Every poset $P$ embeds into the poset $A M_{2}(P(2))$. 


\section{Proof.}

Claim 1. If $y \leq x$ then $(x, 0)$ and $(y, 1)$ are incomparable in $P(2)$. The converse holds if there are two incomparable elements $x^{\prime}, y^{\prime}$ such that $x \leq x^{\prime}$ and $y^{\prime} \leq y$.

If $(x, 0)$ and $(y, 1)$ are comparable then necessarily $(x, 0)<(y, 1)$. In this case there are two incomparable elements $x^{\prime}, y^{\prime}$ such that $x \leq x^{\prime}$ and $y^{\prime} \leq y$. But if $y \leq x$, we get $y^{\prime} \leq x^{\prime}$, a contradiction. Conversely, suppose that $(x, 0)$ and $(y, 1)$ are incomparable. Then clearly, $x$ and $y$ are comparable. Necessarily, $y \leq x$. Otherwise $x<y$. But, from the condition stated, we have $(x, 0) \leq(y, 1)$, a contradiction.

For $x \in P$, set $X_{x}:=\{(x, 0),(x, 1)\}$.

Claim 2. $X_{x} \in A M_{2}(P(2))$.

The set $X_{x}$ is an antichain according to Claim 1. Moreover, every element $\left(x^{\prime}, i^{\prime}\right)$ different from $(x, 0)$ and $(x, 1)$ is comparable to one of these two elements. Indeed, if $x^{\prime}$ is comparable to $x$, then $\left(x^{\prime}, i^{\prime}\right)$ is comparable to $\left(x, i^{\prime}\right)$. If $x^{\prime}$ is incomparable to $x$ then $\left(x^{\prime}, i^{\prime}\right)$ is comparable to $\left(x, 1-i^{\prime}\right)$. This proves that $X_{x}$ is maximal.

Claim 3. The map $x \rightarrow X_{x}$ is an embedding of $P$ into $A M_{2}(P(2))$. That is:

$$
x \leq y \Longleftrightarrow X_{x} \leq X_{y}
$$

Suppose $x \leq y$. Then we have $(x, 0) \leq(y, 0)$ and $(x, 1) \leq(y, 1)$ proving $X_{x} \leq X_{y}$. Conversely, suppose $X_{x} \leq X_{y}$, that is $(x, 0) \leq(y, i)$ and $(x, 1) \leq(y, j)$ for some $i, j \in$ $\{0,1\}$. Due to our ordering, we have $j=1$, hence $x \leq y$ as required.

With this construction, a poset $P$ which is not $\alpha$-bqo but is $\beta$-bqo for every $\beta<\alpha$ leads to a poset $P^{\prime}$ having the same property and for which neither $A M_{2}\left(P^{\prime}\right)$ nor $\bigcup A M_{2}\left(P^{\prime}\right)$ is $\alpha$-bqo. The simplest example of this situation is given in Subsection 6.2.

Theorem 6.3 Let $P$ be a poset with no infinite antichain, and $\alpha$ be a denumerable ordinal, then $A M_{2}(P)$ is $\alpha$-bqo if and only if $\bigcup A M_{2}(P)$ is $\alpha$-bqo.

Proof. If $Q:=\bigcup A M_{2}(P)$ is $\alpha$-bqo then $A(Q)$ is $\alpha$-bqo according to Lemma 2.5 item (e). In particular, $A M_{2}(Q)$ is $\alpha$-bqo. This set includes $A M_{2}(P)$ and the conclusion follows.

For the converse, we prove a bit more. Let $\mathcal{P}$ be a subset of $[P]^{2}$. We quasi-order $\mathcal{P}$ as follows: $X \leq Y$ if for every $x \in X$ there is some $y \in Y$ such that $x \leq y$ and for every $y \in Y$ there is some $x \in X$ such that $x \leq y$.

Let $T$ be a subset of $P^{\prime}:=\bigcup \mathcal{P}$.

Claim If $\mathcal{P}$ is $\alpha$-bqo then $T$ is $\alpha$-bqo.

Let $f: B \rightarrow T$ be a map from a barrier $B$ of type at most $\alpha$ into $T$. For each $s \in B$, select a map $F(s): 2:=\{0,1\} \rightarrow P$ such that $f(s) \in \operatorname{rg}(F(s)) \in \mathcal{P}$. For $s \in B$, set $p(s):=i$ if $F(s)(i)=f(s)$ and for $(s, t) \in B \times B$, set $\rho_{(s, t)}:=\{(i, j) \in 2 \times 2: F(s)(i) \leq F(t)(j)\}$. Note that since an order is transitive, for $s, t, u \in B$ the composition of relations satisfies

$$
\rho_{(t, u)} \circ \rho_{(s, t)} \subseteq \rho_{(s, u)}
$$

Subclaim 1 We may suppose that:

1. $p(s)=i_{0}$ for all $s \in B$ and some $i_{0} \in 2$; 
2. $\rho_{(s, t)}=\rho$ for all pairs $(s, t) \in B \times B$ such that $s \triangleleft t$ and some $\rho \subseteq 2 \times 2$;

3. for every $i \in 2$ there are some $j, j^{\prime} \in 2$ such that $(i, j),\left(j^{\prime}, i\right) \in \rho$.

Proof of Subclaim 1 Since the map $p$ takes only two values, we get from the partition theorem of Nash-Williams $p$ is constant on a subbarrier of $B$. With no loss of generality, we may suppose that this barrier is $B$ proving that 1 holds. Similarly, the map which associate $\rho_{(s, t)}$ to each element $s \cup t \in B^{2}$ takes only finitely many values hence, by the same token, this map is constant on a subbarrier $C$ of $B^{2}$. Necessarily $C=B^{2} \cap[X]^{<\omega}$ for some $X \subseteq \bigcup B$. For $B^{\prime}:=B \cap[X]^{<\omega}$ the condition stated in 2 holds. We may suppose $B^{\prime}=B$. Finally, since $\mathcal{P}$ is $\alpha$-bqo, the map which associates $r g(F(s))$ to $s \in B$ cannot be bad. According to the partition theorem of Nash-Williams this map is perfect on a subbarrier. With no loss of generality, we may suppose this subbarrier equals to $B$. From this 3 follows.

We will prove that $\rho$ is reflexive. With conditions 1 and 2 it follows that $f$ is perfect, proving our claim (indeed, let $s \triangleleft t$. From $1, f(s)=F(s)\left(i_{0}\right)$ and $f(t)=F(t)\left(i_{0}\right)$, from 2 $\rho_{(s, t)}=\rho$. The reflexivity of $\rho$ insures that $\left(i_{0}, i_{0}\right) \in \rho$ that is $\left(i_{0}, i_{0}\right) \in \rho_{(s, t)}$ which amounts to $F(s)\left(i_{0}\right) \leq F(t)\left(i_{0}\right)$. This yields $f(s) \leq f(t)$ as required).

If $\rho$ is not reflexive, then it follows from condition 3 that $\{(0,1),(1,0)\} \subseteq \rho$. From now on, we will suppose this later condition fulfilled.

We say that two elements $s_{0}, s_{1} \in B$ are intertwined and we set $s_{0} \triangleleft_{\frac{1}{2}} s_{1}$ if there is an infinite sequence $X:=a_{0}<\ldots a_{n}<\ldots$ of elements of $\bigcup B$ such that $s_{0}<_{\text {init }} X_{\text {even }}$ and $s_{1}<_{\text {init }} X_{\text {odd }}$, where $X_{\text {even }}:=a_{0}<\ldots a_{2 n}<\ldots$ and $X_{\text {odd }}:=a_{1}<\ldots a_{2 n+1}<\ldots$ We set $B^{\left(\frac{1}{2}\right)}:=\left\{\left(s_{0}, s_{1}\right): s_{0} \triangleleft_{\frac{1}{2}} s_{1}\right\}$ and $B^{\frac{1}{2}}:=\left\{s_{0} \cup s_{1}:\left(s_{0}, s_{1}\right) \in B^{\left(\frac{1}{2}\right)}\right\}$ where $s_{0} \cup s_{1}$ denotes the sequence $w$ whose range is the union of the ranges of $s_{0}$ and $s_{1}$.

We note that

1. if $w \in B^{\frac{1}{2}}$ then the pair $\left(s_{0}, s_{1}\right) \in B^{\left(\frac{1}{2}\right)}$ such that $w=s_{0} \cup s_{1}$ is unique;

2. $B^{\frac{1}{2}}$ is a thin block;

3. if $X:=a_{0}<\ldots a_{n}<\ldots$ is an infinite sequence of elements of $\bigcup B, Y:={ }_{*} X$, $s_{0}, s_{1}, s_{2} \in B$ such that $s_{0}<_{\text {init }} X_{\text {even }}, s_{1}<_{\text {init }} X_{\text {odd }}, s_{2}<_{\text {init }} Y_{\text {odd }}$ then $s_{0} \triangleleft_{\frac{1}{2}} s_{1} \triangleleft_{\frac{1}{2}} s_{2}$ and $s_{0} \triangleleft s_{2}$.

Let $w:=s_{0} \cup s_{1}, w^{\prime}:=s_{0}^{\prime} \cup s_{1}^{\prime} \in B^{\frac{1}{2}}$. We say that $w$ and $w^{\prime}$ are equivalent if there is a map $g$ from $\bigcup\left\{r g\left(F\left(s_{i}\right)\right): i<2\right\}$ onto $\bigcup\left\{r g\left(F\left(s_{i}^{\prime}\right)\right): i<2\right\}$ such that

1. $g \circ\left(F\left(s_{i}\right)\right)=F\left(s_{i}^{\prime}\right)$ for $i<2$;

2. $\rho_{\left(s_{i}, s_{j}\right)}=\rho_{\left(s_{i}^{\prime}, s_{j}^{\prime}\right)}$ for all $i, j<2$;

As one can check easily, this is an equivalence relation on $B^{\frac{1}{2}}$. Furthermore, the number of equivalence classes is finite (one can code each equivalence class by a relational structure on a set of at most 4 elements, this structure been made of four binary relations and 
four unary relations). Since $B^{\frac{1}{2}}$ is a block, it follows from the partition theorem of NashWilliams that one class contains a barrier. Let $C$ be such a barrier, $X \subseteq \cup B$ such that $C:=B^{\frac{1}{2}} \cap[X]^{<\omega}$ and let $B^{\prime}:=B \cap[X]^{<\omega}$. For $s_{0}, s_{1} \in B^{\prime}$ such that $s_{0} \triangleleft_{\frac{1}{2}} s_{1}, \rho_{s_{0}, s_{1}}$ and $\rho_{s_{1}, s_{0}}$ are constant; let $\rho_{\frac{1}{2}}$ and $\rho_{\frac{1}{2}}^{\prime}$ their common value.

For the proof of the next subclaims, we select $s_{0}, s_{1}, s_{2} \in B^{\prime}$ such that $s_{0} \triangleleft_{\frac{1}{2}} s_{1} \triangleleft_{\frac{1}{2}} s_{2}$ and $s_{0} \triangleleft s_{2}$; according to condition 3 above this is possible.

Subclaim $2 \rho_{\frac{1}{2}} \circ \rho_{\frac{1}{2}} \subseteq \rho$

Proof of Subclaim 2 We have $\rho_{\frac{1}{2}}=\rho_{s_{0}, s_{1}}=\rho_{s_{1}, s_{2}}$ and $\rho=\rho_{s_{0}, s_{2}}$. The claimed inclusion follows from Inclusion (4).

Subclaim $3 \rho_{\frac{1}{2}}^{\prime}=\emptyset$

Proof of Subclaim 3 Suppose the contrary; let $(i, j) \in \rho_{\frac{1}{2}}^{\prime}$. Case 1 . $i=j$. Let $k \neq i$. We have $(k, i) \in \rho=\rho_{s_{0}, s_{2}}$ and $(i, i) \in \rho_{\frac{1}{2}}^{\prime}=\rho_{s_{2}, s_{1}}=\rho_{s_{1}, s_{0}}$. By composing these relations, we get with (4) $(k, i) \in \rho_{s_{0}, s_{0}}$ contradicting the fact that $r g\left(F\left(s_{0}\right)\right)$ is an antichain. Case 2 . $i \neq j$. then from $(i, j) \in \rho_{\frac{1}{2}}^{\prime}=\rho_{s_{2}, s_{1}}=\rho_{s_{1}, s_{0}}$ and $(j, i) \in \rho=\rho_{s_{0}, s_{2}}$ we get, by composing these relations, $(i, j) \in \rho_{s_{1}, s_{1}}$ contradicting the fact that $\operatorname{rg}\left(F\left(s_{1}\right)\right)$ is an antichain and proving Subclaim 3.

Subclaim $4 \rho_{\frac{1}{2}}$ satisfies condition 3 of Subclaim 1 .

Proof of Subclaim 4 Since $r g\left(F\left(s_{0}\right)\right)$ and $r g\left(F\left(s_{1}\right)\right)$ are two maximal antichains, each element of one is comparable to some element of the other. Since $\rho_{s_{1}, s_{0}}=\rho_{\frac{1}{2}}^{\prime}=\emptyset$, $r g\left(F\left(s_{0}\right)\right) \leq r g\left(F\left(s_{1}\right)\right)$ and the result follows.

Now, if $\rho_{\frac{1}{2}}$ is reflexive, it follows from Subclaim 2 that $\rho$ is reflexive and our claim is proved. If $\rho_{\frac{1}{2}}$ is not reflexive then from Subclaim 4 it follows that $\{(0,1),(1,0)\} \subseteq \rho_{\frac{1}{2}}$. With Subclaim 2 this yields $(0,0),(1,1) \in \rho$ that is $\rho$ is reflexive and the proof of our claim is complete.

\subsection{Rado's poset}

Let $V:=\left\{(m, n) \in \mathbb{N}^{2}: m<n\right\}$. We denote by $\leq_{R}$ the following relation on $V$ :

$$
(m, n) \leq_{R}\left(m^{\prime}, n^{\prime}\right) \text { if either } m=m^{\prime} \text { and } n \leq n^{\prime} \text { or } n<m^{\prime}
$$

This relation is an order. We denote by $R$ the resulting poset. This poset, discovered by R. Rado [24], is at the root of the discovery of bqo's. R. Rado observed that $R$ is wqo but $I(R)$ is not wqo and has shown that a poset $P$ is $\omega^{2}$-bqo if and only if $I(P)$ is wqo. R. Laver [13] has shown that a poset $P$ which is wqo, and not $\omega^{2}$-bqo contains a copy of $R$. Applying the construction given in Lemma 6.2 we have:

Lemma 6.4 The poset $A M_{2}(R(2))$ is wqo but not $\omega^{2}$-bqo.

Proof. As a union of two wqo posets, $R(2)$ is wqo. Hence $A M(R(2))$ is wqo for the domination order. In particular $A M_{2}(R(2))$ is wqo. Since $A M_{2}(R(2))$ embeds $R$, it cannot be $\omega^{2}$-bqo. 
Lemma 6.5 $\bigcup A M_{m}(R)$ is bqo for every integer $m$ and $R$ embeds into $A M(R)$.

\section{Proof.}

a) $\bigcup A M_{m}(R)$ is bqo. Let $m<\omega$. Then $\bigcup A M_{m}(R) \subseteq\{(i, j): i<m, i<j<\omega\}$. Indeed, let $A \in A M_{m}(R)$. Then we claim that for each $i<m$ there is some $(i, j) \in A$ with $i<j$. Consequently $\bigcup A M_{m}(R)$ is bqo.

If there is $i<m$ so that there is no $(i, j) \in A$ with $i<j$ then: Let $i_{0}<m$ be least such that $\left(i_{0}, j\right) \notin A$ for all $j$. Since if $(i, j),\left(i^{\prime}, j^{\prime}\right) \in A$ then $i \neq i^{\prime}$ we have $k:=\max \{i$ : there exists a $j$ with $(i, j) \in A\} \geq m$. Let $h$ be such that $(k, h) \in A$. There exists $(i, j) \in A$ comparable with $\left(i_{0}, k\right)$. If $(i, j) \leq_{R}\left(i_{0}, k\right)$ then $j<i_{0}<k$ and hence $(i, j) \leq_{R}(k, h)$, against $A$ being an antichain. If $\left(i_{0}, k\right) \leq_{R}(i, j)$ then $k<i$ against the definition of $k$.

b) $R$ embeds into $A M(R)$. Since $R$ is not $\omega^{2}$ bqo, $A M(R)$ is not $\omega^{2}$ bqo (Theorem 5.5). Hence from Laver's result mentioned above, the poset $R$ embeds into $A M(R)$. For the sake of simplicity we indicate a direct proof.

Let $t: R \rightarrow R$ be defined by $t(m, n):=(2 m, 2 n+1)$ and let $\Psi: R \rightarrow A M(R)$ be defined by $\Psi(m, n):=\varphi(t(m, n))$. Hence $\Psi(m, n)$ is the least maximal antichain of $R$ containing $t(m, n)$, see Lemma 5.3. Note that $t$ is an order preserving embedding of $R$ to $R$ and that $\varphi$ is order preserving. Hence $\Psi$ is order preserving.

\subsection{Three element maximal antichains}

Lemma 6.6 Let $P:=(V ; \leq)$ be a poset. Let $L:=(V ; \sqsubseteq)$ be a linear extension of $P$ with the property that if $x<y$ then there is $a z$ with $x \sqsubset z \sqsubset y$ and $z$ is incomparably in $P$ to both $x$ and $y$. Then there is a poset $Q$ which is a union of a copy of $P$ and two copies of $L$ for which $\bigcup A M_{3}(Q)=Q$ and $A M_{3}(Q)$ is isomorphic to $L$.

Proof. On $V \times 3$ define the following strict order relation $<_{Q}$ :

$$
(x, i)<_{Q}(y, j) \text { if } \begin{cases}i=j=1 & \text { and } x<y \text {, or } \\ 1 \neq i \text { or } j \neq 1 & \text { and } i \leq j \text { and } x \sqsubset y .\end{cases}
$$

Let $Q=\left(V \times 3 ; \leq_{Q}\right)$ be the resulting poset by adding the identity relation to $<_{Q}$. The order induced by $\leq_{Q}$ on $V \times\{i\}$ coincides with the order $\leq$ on $V$ if $i=1$, whereas it coincides with $\sqsubseteq$ if $i \neq 1$.

Let $A:=\left\{\left(x_{0}, i_{0}\right),\left(x_{1}, i_{1}\right),\left(x_{2}, i_{2}\right), \ldots,\left(x_{n-1}, i_{n-1}\right)\right\}$ be a finite antichain of $Q$ with $x_{0} \sqsubseteq$ $x_{1} \sqsubseteq x_{2} \sqsubseteq x_{3} \sqsubseteq \cdots \sqsubseteq x_{n-1}$. If $i_{j} \neq 2$ for any $j \in n$ then $\left\{\left(x_{0}, 2\right)\right\} \cup A$ is an antichain of $Q$. If $i_{j} \neq 0$ for any $j \in n$ then $\left\{\left(x_{n-1}, 0\right)\right\} \cup A$ is an antichain of $Q$. It follows that every element of $A M_{3}(Q)$ is of the form $\left\{\left(x_{0}, 0\right),\left(x_{1}, 1\right),\left(x_{2}, 2\right)\right\}$ with $x_{0} \sqsupseteq x_{1} \sqsupseteq x_{2}$.

Let $A:=\left\{\left(x_{0}, 0\right),\left(x_{1}, 1\right),\left(x_{2}, 2\right)\right\} \in A M_{3}(Q)$. Assume for a contradiction that $x_{0} \neq x_{1}$. Because $A$ is a maximal antichain it follows that $x_{1}<x_{0}$. According to the assumptions of the Lemma, there exists an element $y \in V$ which is not related to $x_{1}$ and $x_{0}$ and with $x_{1} \sqsubset y \sqsubset x_{0}$. Then $\left\{\left(x_{0}, 0\right),(y, 1),\left(x_{1}, 1\right),\left(x_{2}, 2\right)\right\}$ is an antichain. In a similar way we obtain that $x_{2}=x_{1}$. It follows that $A M_{3}(Q)=\{\{y\} \times 3: y \in V\}$.

We conclude that $A M_{3}(Q)$ is isomorphic to $L$ and $\bigcup A M_{3}(Q)=\mathrm{Q}$. 
Corollary 6.7 There exists a poset $Q$ for which $A M_{3}(Q)$ is bqo but $\bigcup A M_{3}(Q)$ is not bqo.

Proof. A poset $P$ which is wqo and not bqo but satisfies the conditions of Lemma 6.6 leads to a poset $Q$ which is wqo, not bqo, and for which $A M_{3}(Q)$ is well-ordered (because every linear extension of a wqo is a well-ordering) and hence bqo, but $\bigcup A M_{3}(Q)$ is not bqo. One may take for $P$ Rado's example and for $L$ the lexicographic order defined by $(m, n) \sqsubseteq\left(m^{\prime}, n^{\prime}\right)$ iff $n<n^{\prime}$ or $n=n^{\prime}$ and $m \geq m^{\prime}$.

\section{Notation, basic definitions and facts}

Let $P$ denote a partially ordered set.

Poset, qoset, chain, well-founded, wqo, well-ordered:

If $(P ; \leq)$ is a partially ordered set, a poset, we will often just write $P$ for $(P ; \leq)$. We write $a \leq b$ for $(a, b) \in \leq$. A qoset is a quasi ordered set and a linearly ordered poset is a chain.

A qoset $P$ is well-founded if it contains no infinite descending chain

$$
\cdots<x_{n}<\cdots<x_{0}
$$

and if in addition, $P$ contains no infinite antichain then it is well-quasi-ordered (wqo). If $P$ is a chain and well-quasi-ordered then it is well-ordered.

Initial segment, principal, $\mathbf{I}(P), \mathbf{I}_{<\omega}(P), \downarrow X$ :

A subset $I$ of $P$ is an initial segment (or is closed downward) if $x \leq y$ and $y \in I$ imply $x \in I$. We denote by $\mathbf{I}(P)$ the set of initial segments of $P$ ordered by inclusion.

Let $X$ be a subset of $P$, then:

$$
\downarrow X:=\{y \in P: y \leq x \text { for some } x \in X\} .
$$

We say that $\downarrow X$ is generated by $X$. If $X$ contains only one element $x$, we write $\downarrow x$ instead of $\downarrow\{x\}$. An initial segment generated by a singleton is principal and it is finitely generated if it is generated by a finite subset of $P$. We denote by $\mathbf{I}_{<\omega}(P)$ the set of finitely generated initial segments.

up $(P), \operatorname{down}(P)$

We set $u p(P):=\{\uparrow x: x \in P\}$ and $\operatorname{down}(P):=\{\downarrow x: x \in P\}$.

Tailalg $(P)$, Taillat $(P)$.

The tail algebra of $P$ is the subalgebra Tailalg $(P)$ of the Boolean algebra $(\mathfrak{P}(P), \cap, \cup$, $\backslash, \emptyset, P)$ generated by up $(P)$, (See [17], Chapter 2, page 40). The tail lattice of $P$ is the bounded sublattice Taillat $(P)$ of $(\mathfrak{P}(P), \cap, \cup, \emptyset, P)$ generated by up $(P)$. 
$\leq_{\text {dom }}$, domination quasiorder:

A subset $X$ of $P$ is being dominated by the subset $Y$ of $P, X \leq_{\text {dom }} Y$, if for every $x \in X$ there is a $y \in Y$ such that $x \leq y$. The domination relation is a quasi-order on the powerset $\mathfrak{P}(P)$. The resulting ordered set is isomorphic to $\mathbf{I}(P)$, ordered by inclusion, via the map which associates with $X \in \mathfrak{P}(P)$ the initial segment $\downarrow X$.

$S_{\omega}(P)$, strictly increasing sequence:

A sequence $\left(x_{n}\right)_{n<\omega}$ of elements of $P$ is strictly increasing if

$$
x_{0}<x_{1} \cdots<x_{n}<x_{n+1}<\cdots
$$

We denote by $S_{\omega}(P)$ the set of strictly increasing sequences of elements of $P$. For $\left(x_{n}\right)_{n<\omega} \in S_{\omega}(P)$ and $\left(y_{n}\right)_{n<\omega} \in S_{\omega}(P)$ we set $\left(x_{n}\right)_{n<\omega} \leq_{d o m}\left(y_{n}\right)_{n<\omega}$ if for every $n<\omega$ there is some $m<\omega$ such that $x_{n} \leq y_{m}$. This defines a quasi-order on $S_{\omega}(P)$. If we identify each $\left(x_{n}\right)_{n<\omega} \in S_{\omega}(P)$ with the subset $\left\{x_{n}: n<\omega\right\}$ of $P$, this quasi-order is induced by the domination relation on subsets. Which explains the notational use.

$\mathcal{J}(P), \mathcal{J}^{\neg \downarrow}(P)$, ideal, non principal ideal:

An ideal of $P$ is a non empty initial segment $I$ which is up-directed, that is every pair $x, y \in I$ has an upper bound $z \in I$. Its cofinality, $c f(I)$, is the least cardinal $\kappa$ such that there is some set $X$ of size $\kappa$ such that $I=\downarrow X$. The cofinality of $I$ is either 1 , in which case it has a largest element and is said to be principal, or is infinite. We denote by $\mathcal{J}^{\neg}(P)$ the set of non principal ideals of $P$.

Note the following fact, which goes back to Erdős-Tarski [5], (see [6]):

Fact 7.1 A poset $P$ has no infinite antichain if and only if every initial segment of $P$ is a finite union of ideals.

\section{$P^{\text {dual }}, \mathbf{F}(P), \mathbf{F}_{<\omega}(P), \mathcal{F}(P)$, filter:}

The dual of $P$ is the poset obtained from $P$ by reversing the order; we denote it by $P^{d u a l}$. A subset which is respectively an initial segment, a finitely generated initial segment or an ideal of $P^{\text {dual }}$ will be called a final segment, a finitely generated final segment or a filter of $P$. We denote by $\mathbf{F}(P), \mathbf{F}_{<\omega}(P)$, and $\mathcal{F}(P)$ respectively, the collection of final segments, finitely generated final segments, and filters of $P$ ordered by inclusion.

$$
\underline{\mathfrak{P}(P), \mathfrak{P}_{\leq \omega}(P), \mathfrak{P}_{<\omega}(P), A(P), A M(P), A M_{n}(P):}
$$

$\mathfrak{P}(P)$ denotes the set of subsets of $P . \mathfrak{P}_{\leq \omega}(P)$ the set of countable subsets of $\mathfrak{P}$. $\mathfrak{P}_{<\omega}(P)$ the set of finite subsets of $\mathfrak{P}(P) . A(P)$ is the collection of antichains of $P$ and $A M(P)$ the collection of maximal antichains of $P$. The quasi-order of domination defined on $\mathfrak{P}(P)$ induces an ordering on the set $A(P)$ of antichains of $P$. The sets $A(P)$ and $A\left(P^{\text {dual }}\right)$ are equal. We may order $A(P)$ by the dual of the domination order of $P^{d u a l}$. In general this order is different from the domination order on $A M(P)$. These two orders coincide on $A M(P)$. We denote by $A M_{n}(P)$ the collection of $n$-element maximal antichains of $P$. 
$\leq_{\text {succ }}, \leq_{\text {pred }}, \leq_{\text {crit }}:$

Let $P$ be a poset. We write $a \leq_{\operatorname{pred}(P)} b$ if $x<a$ implies $x<b$ for every $x \in P$. We write $a \leq_{\text {succ }(P)} b$ if $b<y$ implies $a<y$ for every $y \in P$. We write $a \leq_{\text {prec }} b$, or $a \leq_{\text {succ }} b$, if $P$ is understood. We denote by $\leq_{\text {crit }}$ the intersection of the quasi-orderings $\leq_{\text {succ }}$ and $\leq_{\text {pred }}$. We denote by $\left(P ; \leq_{\text {prec }}\right),\left(P ; \leq_{\text {succ }}\right)$ and $\left(P ; \leq_{\text {pred }}\right)$ the corresponding quasi-ordered sets.

A pair $(a, b)$ of elements of $P$ is critical if $a$ and $b$ are incomparable, $a \leq_{\operatorname{crit}(P)} b$ and $a \leq_{\operatorname{succ}(P)} b$.

\section{Interval order:}

The poset $P$ is an interval-order if $P$ is isomorphic to a subset $\mathcal{J}$ of the set $\operatorname{Int}(C)$ of non-empty intervals of some chain $C$. The intervals are ordered as follows: for every $I, J \in \operatorname{Int}(C), I<J$ if $x<y$ for every $x \in I$, every $y \in J$.

Interval orders have neat characterizations in different ways: maximal antichains, associated preorders or obstructions, see [7], [26]. We recall this important characterization:

Theorem 7.2 The following properties are equivalent:

(i) $P$ is an interval order.

(ii) $\left(P ; \leq_{\text {pred }}\right)$ is a total qoset.

(iii) $\left(P ; \leq_{\text {succ }}\right)$ is a total qoset.

(iv) $P$ does not contain a subset isomorphic to $\underline{2} \oplus \underline{2}$, the direct sum of two copies of the two-element chain.

(v) $A M(P)$ is a chain.

$\mathbb{N},[X]^{<\omega}, l(s), \lambda(s),{ }_{*} s, s_{*}, s \cdot t, s \leq_{\text {end }} t, s \leq_{\text {in }} t, s \leq_{\text {lex }} t, s \triangleleft t:$

The set of non-negative integers is denoted by $\mathbb{N}$, the set of $n$-element subsets of $X \subseteq \mathbb{N}$ by $[X]^{n}$ and the set of finite subsets of $X \subseteq \mathbb{N}$ by $[X]^{<\omega}$. We identify each member $s$ of $[\mathbb{N}]^{<\omega}$ with a strictly increasing sequence, namely the list of its elements written in an increasing order, eg $\{3,4,8\}$. Let $s \in[\mathbb{N}]^{<\omega}$; the length of $s, l(s)$, is the number of its elements. For $m:=l(s) \neq 0$, we write $s:=\{s(0), \ldots, s(m-1)\}$ with $s(0)<s(1)<\cdots<s(m-1)$. The smallest element of $s$ is $s(0)$ the largest, denoted by $\lambda(s)$, is $s(m-1)$.

We denote by ${ }_{*} s$ the sequence obtained from $s$ by deleting its first element and by $s_{*}$ the sequence obtained by deleting the last element. (With the convention that ${ }_{*} \emptyset=\emptyset_{*}=\emptyset$.) We denote by $(a)$ the one element sequence with entry $a$.

Let $s, t \in[\mathbb{N}]^{<\omega}$. If $\lambda(s)<t(0)$ then $s \cdot t$ is the concatenation of $s$ and $t$. Set $s \leq_{\text {end }} t$ if $\lambda(s) \leq \lambda(t)$ and $s_{*}=t_{*}$. We denote by $s \leq_{i n} t$ the fact that $s$ is an initial segment of $t$ and by $s \leq_{l e x} t$ the fact that $s$ is smaller than $t$ in the lexicographic order. (For example $\{3,5,8,9\} \leq_{\text {lex }}\{3,5,9,15\}$.)

If there exists an $r \in[\mathbb{N}]^{<\omega}$ with $s<_{i n} r$ and $t={ }_{*} r$ then $s \triangleleft t$. For example $\{i\} \triangleleft\{j\}$ if and only if $i<j,(r=\{i, j\})$; also $\{i, j\} \triangleleft\left\{i^{\prime}, j^{\prime}\right\}$ if and only if $j=i^{\prime}$ and then $r=\left\{i, i^{\prime}, j^{\prime}\right\}$. 
$\underline{\cup B, B_{\lceil X} \text {, block, thin block, barrier, end-closed barrier }}$

Let $B \subseteq[\mathbb{N}]^{<\omega}$ and $X \subseteq \mathbb{N}$. We will denote by $\bigcup B$ the union of the elements of $B$ and let $B_{\mid X}:=B \cap[X]^{<\omega}$. The set $B$ is a block ${ }^{1}$ if:

1. $B$ is infinite.

2. For every infinite subset $X \subset \bigcup B$ there is some $s \in B \backslash\{\emptyset\}$ such that $s \leq_{\text {in }} X$.

If $B$ is a block and an antichain for the order $\leq_{i n}$ then $B$ is a thin block, whereas $B$ is a barrier if it is a block and an antichain for the inclusion order. A typical barrier is the set $[\mathbb{N}]^{n}$ of $n$-element subsets of $\mathbb{N}$.

Trivially, every block contains a thin block, the set $\min _{\leq_{i n}}(B)$ of $\leq_{\text {in }}$ minimal elements of the block. Moreover, if $B$ is a block, resp. a thin block, and $X$ is an infinite subset of $\bigcup B$ then $B_{\mid X}$ is a block, resp. a thin block. A barrier $B$ is end-closed if

$$
s \leq_{\text {end }} t \quad \text { and } \quad s \in B \quad \text { implies } \quad t \in B .
$$

$\underline{T(B),{ }_{*} B, B_{s},{ }_{s} B, B_{*}, B^{2}, B^{\circ}, B \leq_{\text {in }} B^{\prime}:}$

Let $B, B^{\prime} \subseteq[\mathbb{N}]^{\omega}$.

$\mathrm{T}(B):=\left(\left\{t: \exists s \in B\left(t \leq_{\text {in }} s\right)\right\}, \leq_{i n}\right)$.

Let for $s \in[\mathbb{N}]^{<\omega}$ :

$B_{s}:=\left\{t \in B: s \leq_{\text {in }} t\right\}$.

${ }_{s} B:=\left\{t \in[\mathbb{N}]^{<\omega}: s \cdot t \in B\right\}=\left\{r \backslash s: r \in B_{s}\right\}$. Note that if $B$ is a thin block and ${ }_{s} B$ is non-empty then it is a thin block.

${ }_{*} B:=\left\{{ }_{*} s: s \in B\right\}$.

$B_{*}:=\left\{s_{*}: s \in B\right\}$.

$B^{2}:=\{u:=s \cup t: s, t \in B$ and $s \triangleleft t\}$. (This despite the possible confusion with the cartesian square of $B$.)

$B^{\prime} \leq_{i n} B$ if for every $s^{\prime} \in B^{\prime}$ there is some $s \in B$ such that $s^{\prime} \leq_{i n} s$. This is the quasi-order of domination associated with the order $\leq_{\text {in }}$ on $[\mathbb{N}]^{<\omega}$.

bqo, good, bad

A map $f$ from a barrier $B$ into a poset $P$ is good if there are $s, t \in B$ with $s \triangleleft t$ and $f(s) \leq f(t)$. Otherwise $f$ is bad.

Let $\alpha$ be a denumerable ordinal. A poset $P$ is $\alpha$-better-quasi-ordered if every map $f$ : $B \rightarrow P$, where $B$ is a barrier of order type at most $\alpha$, is good.

A poset $P$ is better-quasi-ordered if it is $\alpha$-better-quasi-ordered for every denumerable ordinal $\alpha$.

\footnotetext{
${ }^{1}$ We stick to the definition of Nash-Williams, 1968 [19]; in some papers, a block is what we call a thin block.
} 


\section{References}

[1] M. Assous, Caractérisation du type d'ordre des barrières de Nash-Williams. Publ. Dép. Math. (Lyon) 11 (1974), no. 4, 89-106.

[2] G.Behrendt, Maximal antichains in partially ordered sets. Eleventh British Combinatorial Conference (London, 1987). Ars Combin. 25 (1988), C, 149-157.

[3] M.Bekkali, M.Pouzet, D.Zhani, Incidence structures and Stone-Priestley duality, 2004, see arXiv:math.CO/0601121 v1 6Jan 2006.

[4] R.Bonnet, Private communication, december 2004.

[5] P.Erdős, A.Tarski, On families of mutually exclusive sets, Annals of Math. 44(1943), 315-329.

[6] R. Fraïssé, Theory of relations. North-Holland Publishing Co., Amsterdam, 2000.

[7] P.C. Fishburn, Interval Orders and Interval graphs, Wiley, 1985.

[8] F. Galvin, A generalization of Ramseys theorem. Notices Amer. Math. Soc. vol.15 p.548 (1969) Abstract 68 T 368.

[9] G. Grätzer, General lattice theory. Second edition. New appendices by the author with B. A. Davey, R. Freese, B. Ganter, M. Greferath, P. Jipsen, H. A. Priestley, H. Rose, E. T. Schmidt, S. E. Schmidt, F. Wehrung and R. Wille. Birkhuser Verlag, Basel, 1998. xx+663 pp.

[10] G. Higman, Ordering by divisibility in abstract algebras, Proc. London. Math. Soc. 2 (3), (1952), 326-336.

[11] J. B. Kruskal, Well-quasi-ordering, the Tree Theorem, and Vazsonyi's conjecture. Trans. Amer. Math. Soc., 95 (1960)210-225.

[12] J. B. Kruskal, The theory of well-quasi-ordering : a frequently discovered concept. J. Com. Th., 13:297-305, 1972.

[13] R. Laver, Well-quasi-orderings and sets of finite sequences. Math. Proc. Cambridge Philos. Soc. 79 (1976), no. 1, 1-10.

[14] A.Marcone, Foundations of bqo theory Trans. Amer. Math. Soc., 345 (1994), 641-660

[15] A.Marcone, Fine analysis of the quasi-orderings on the power set, Order, 18, (2001)339-347.

[16] E. C. Milner, Basic wqo- and bqo-theory, In Graphs and order (Banff, Alta., 1984), pages 487-502, Reidel, Dordrecht, 1985.

[17] G.D. Monk, Cardinal invariants on Boolean algebras, In Progress in Mathematics, Vol. 142, Birkhäuser, Basel, 1996.

[18] C. St. J. A. Nash-Williams, On well-quasi-ordering finite trees. Proc. Cambridge Philos. Soc., 59:833-835, 1963.

[19] C. St. J. A. Nash-Williams, On better-quasi-ordering transfinite sequences. Proc. Cambridge Philos. Soc., 64:273-290, 1968.

[20] M.Pouzet, Sur les prémeilleurordres, Ann. Inst. Fourier(Grenoble) 22(1972)1-20. 
[21] M. Pouzet, Sur la théorie des relations. Thèse d'état, Université Claude-Bernard, Lyon 1, 1978.

[22] M.Pouzet, Graphs and posets with no infinite independent set. Finite and infinite combinatorics in sets and logic (Banff, AB, 1991), 313-335, NATO Adv. Sci. Inst. Ser. C Math. Phys. Sci., 411, Kluwer Acad. Publ., Dordrecht, 1993.

[23] F. P. Ramsey, On a problem of formal logic, Proc. London Math. Soc., 30, (1930), 264-286.

[24] R.Rado, Partial well-ordering of a set of vectors, Mathematika, 1 (1954), 89-95.

[25] N.Robertson, P. Seymour, Graph minors - a survey. Surveys in combinatorics 1985 (Glasgow, 1985), 153-171, London Math. Soc. Lecture Note Ser., 103, Cambridge Univ. Press, Cambridge, 1985.

[26] M. Wiener, A contribution to the theory of relative position, Proc. Camb. Philos. Soc., 17, 1914, p. 441-449. 